REVIEW ARTICLE

\title{
Order Parameters and Phase Diagrams of Multiferroics
}

\author{
A. B. Harris ${ }^{1}$, Amnon Aharony ${ }^{2}$, and Ora Entin-Wohlman ${ }^{2}$ \\ 1 Department of Physics and Astronomy, University of Pennsylvania, \\ Philadelphia, PA 19104, USA \\ 2 Department of Physics, Ben Gurion University, Beer Sheva 84105 ISRAEL* \\ E-mail: aaharony@bgu.ac.il
}

\begin{abstract}
The symmetry properties, order parameters, and magnetoelectric phase diagrams of multiferroics are discussed. After brief reviews of $\mathrm{Ni}_{3} \mathrm{~V}_{2} \mathrm{O}_{8}$, $\mathrm{TbMnO}_{3}$, and $\mathrm{RbFe}\left(\mathrm{MoO}_{4}\right)_{2}$, we present a detailed analysis of $\mathrm{RMn}_{2} \mathrm{O}_{5}$ (with $\mathrm{R}=\mathrm{Y}, \mathrm{Ho}, \mathrm{Dy}, \mathrm{Er}, \mathrm{Tb}, \mathrm{Tm})$.
\end{abstract}

PACS numbers: $75.25 .+\mathrm{z}, 75.10 . \mathrm{Jm}, 75.40 . \mathrm{Gb}$

Submitted to: J. Phys.: Condens. Matter 
Order Parameters and Phase Diagrams of Multiferroics

\section{INTRODUCTION}

Here we review recent and new developments which elucidate the symmetry and the description of the magnetic and dielectric states of multiferroics using order parameters. After some examples where the magnetoelectric (ME) behavior is relatively simple, we discuss a proposed generic phase diagram for the so-called " 125 " systems, $\mathrm{RMn}_{2} \mathrm{O}_{5}$, where $\mathrm{R}$ is a rare earth. The most important consequence of the phenomenological theories we develop is to provide a general framework for understanding the magnetic and dielectric properties of these materials and how these properties combine to produce the interesting ME phenomena.

Briefly, this article is organized as follows. In Sec. 2 we discuss the characterization of the magnetic structure obtained from symmetry arguments. Here we discuss briefly a simplified version of the group theoretical approach (known as representation theory) which is supplemented by less well-known arguments involving the use of inversion symmetry. As examples we consider $\mathrm{Ni}_{3} \mathrm{~V}_{2} \mathrm{O}_{8}(\mathrm{NVO}), \mathrm{TbMnO}_{3}$, and $\mathrm{RbFe}\left(\mathrm{MoO}_{4}\right)_{2}$ (RFMO) and discuss the introduction of order parameters (OP's) to characterize the magnetic symmetry. We then give a brief review of how symmetry restricts the form of the ME interaction when it is written in terms of both magnetic and dielectric OP's. In Sec. 3 we give a detailed discussion of how these concepts enable us to construct a generic phase diagram for the 125 family of multiferroics, which does not rely on a knowledge of the details of the microscopic interactions. Section 4 contains an application of the theory of critical phenomena to the 125's, and the paper is briefly summarized in section 5 .

\section{SYMMETRY AND MAGNETIC STRUCTURE}

Here we give a simplified review of the role of symmetry in determining the structure of the magnetically ordered phase which develops at a continuous phase transition. This subject is of ancient vintage, being discussed about 60 years ago by Landau (see [1]). However, some reviews which discuss the analysis of diffraction data 2, 3] overlook the importance of inversion symmetry in reducing the number of parameters needed to describe the ordered magnetic structures. For multiferroics this was first corrected quite recently by Lawes et al. [4], by Kenzelmann et al. [5] and in more detail by Harris [6], which we follow here. Formal treatments appeared some time ago [7]. Recent papers include Schweizer et al. 8 , and Radaelli and Chapon $[9]$.

We start by assuming that the paramagnetic phase is characterized by a primitive unit cell with $n_{\tau}$ magnetic sites. The Landau expansion of $F_{2}$, the magnetic free energy at quadratic order in the spin components, is

$$
F_{2}=\sum_{\mathbf{q}} \sum_{\tau, \tau^{\prime}=1}^{n_{\tau}} \sum_{\alpha, \beta}\left[\chi^{-1}(\mathbf{q})\right]_{\tau, \alpha ; \tau^{\prime}, \beta} S_{\alpha}(\mathbf{q}, \tau)^{*} S_{\beta}\left(\mathbf{q}, \tau^{\prime}\right),
$$

where $\chi$ is the wave-vector dependent susceptibility matrix and

$$
S_{\alpha}(\mathbf{R}, \tau)=\sum_{\mathbf{q}} S_{\alpha}(\mathbf{q}, \tau) e^{i \mathbf{q} \cdot \mathbf{R}},
$$

where $S_{\alpha}(\mathbf{R}, \tau)$ is the $\alpha$-component of spin of the $\tau$ th magnetic site in the unit cell at $\mathbf{R}$ and $S_{\alpha}(-\mathbf{q}, \tau)=S_{\alpha}(\mathbf{q}, \tau)^{*}$. For each value of the wave vector the inverse susceptibility has $3 n_{\tau}$ eigenvalues (which may or may not be distinct from one another). At high temperature $T$ all these eigenvalues are positive and the paramagnetic state is 
thermodynamically stable. As $T$ is reduced through a critical value, $T_{c}$, one eigenvalue, $\lambda_{c}\left(\mathbf{q}_{c}\right)$, at some wave vector $\mathbf{q}_{c}$ (and wave vectors equivalent to it by symmetry which comprise the star of $\mathbf{q}_{c}$ ) approaches zero, signaling an instability of the paramagnetic phase to the formation of long-range order at the critical wave vector $\mathbf{q}_{c}$ associated with this critical eigenvalue. The actual value of $\mathbf{q}_{c}$ is determined by the microscopic interactions. Since these interactions are not well known, we regard the wave vector as an experimentally determined parameter. The degeneracy of this critical eigenvalue $\lambda_{c}$ is $n_{q} \mathcal{N}$, where $n_{q}$ is the number of wave vectors in the star of $\mathbf{q}$ and $\mathcal{N}$ is the dimensionality of the irreducible representation (irrep) of the symmetry group (the so-called "little group") which leaves the wave vector invariant. (For ferromagnetic Ising, $x-y$, and Heisenberg models $\mathcal{N}$ assumes the values 1 , 2, and 3, respectively.) To avoid technicalities, in this section we consider the simplest case, $\mathcal{N}=1$. This case is simple because then we can use the familiar principle that the eigenvectors of a matrix (here the inverse susceptibility) are also simultaneously eigenvectors of operators (here the symmetry operations $\mathcal{O}_{i}$ of the space group which leave the selected wave vector invariant) which commute with each other and with the matrix. In this way we avoid using the full apparatus of group theory and the reader need not know anything at all about "irreps". We now illustrate this idea and show how inversion symmetry introduces further simplifications for three recently studied multiferroic magnetic materials, whose lattice structures are shown in figure 1 and whose positions (except for RFMO where the Fe ions form a Bravais lattice) are given in table 10

Figure 1. (Color online). (a) The six Ni sites in the unit cell of NVO. Circles represent "spine" sites and squares represent the "cross-tie" sites. The axis of the two-fold rotation about the $a$-axis is shown. The glide plane is indicated by the mirror plane at $z=\frac{3}{4}$ and the arrow above $m_{c}$ indicates that a translation of $b / 2$ in the $b$-direction is involved. (b) The four Mn sites (small circles) and four $\mathrm{Tb}$ sites (large circles) in the unit cell of $\mathrm{TbMnO}_{3}$. The glide $m_{a}$ is indicated by the mirror plane at $x=\frac{3}{4}$ followed by a translation of $b / 2$ along the $\mathbf{b}$ direction. The planes at $z=1 / 4$ and $z=3 / 4$ are mirror planes. (c) RFMO, where large balls are Fe spins $5 / 2$ on a stacked triangular lattice, small balls are oxygens, Mo ions are inside the oxygen tetrahedra, and the $\mathrm{Rb}$ ions are not shown.
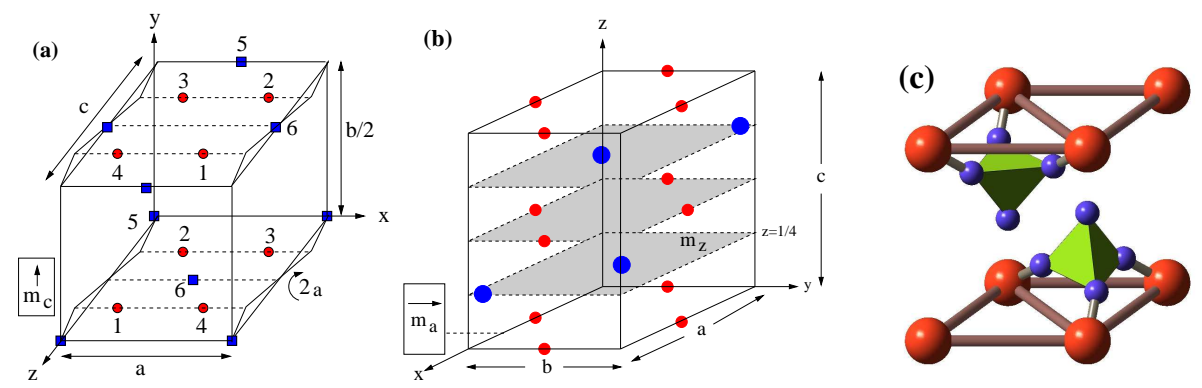

\section{1. $N V O$}

For NVO the incommensurate (IC) wave vector for magnetic ordering is 11, 12] $\mathbf{q} \cong 0.28(2 \pi / a) \hat{\mathbf{a}}$. Thus the space group operations $\mathcal{O}_{i}$ which leave the wave vector invariant are generated by $2_{a}$, a two-fold rotation about the $a$-axis and passes through

$\ddagger$ We interchangeably denote the $\mathbf{a}, \mathbf{b}$, and $\mathbf{c}$ axes as $x, y$, and $z$, respectively. 
Order Parameters and Phase Diagrams of Multiferroics

Table 1. General positions 10 (given as fractions of lattice constants) within the primitive unit cell for NVO (space group Cmca) and $\mathrm{TbMnO}_{3}$ (space group Pbnm). Here $r+\equiv r+1 / 2,2_{\alpha}$ is a two-fold rotation (or screw) axis, and $m_{\alpha}$ is a mirror (or glide).

\begin{tabular}{||c|c||c|c||}
\hline \multicolumn{2}{|c||}{} & \multicolumn{2}{c||}{$\mathrm{Ni}_{3} \mathrm{~V}_{2} \mathrm{O}_{8}$} \\
\hline $\mathrm{E} \mathbf{r}=(x, y, z)$ & $2_{c} \mathbf{r}=(\bar{x}, \bar{y}+, z+)$ & $E \mathbf{r}=(x, y, z)$ & $2_{a} \mathbf{r}=(x+, \bar{y}+, \bar{z})$ \\
$2_{a} \mathbf{r}=(x, \bar{y}, \bar{z})$ & $2_{b} \mathbf{r}=(\bar{x}, y+, \bar{z}+)$ & $2_{c} \mathbf{r}=(\bar{x}, \bar{y}, z+)$ & $2_{b} \mathbf{r}=(\bar{x}+, y+, \bar{z}+)$ \\
$\mathcal{I} \mathbf{r}=(\bar{x}, \bar{y}, \bar{z})$ & $m_{c} \mathbf{r}=(x, y+, \bar{z}+)$ & $\mathcal{I} \mathbf{r}=(\bar{x}, \bar{y}, \bar{z})$ & $m_{a} \mathbf{r}=(\bar{x}+, y+, z)$ \\
$m_{a} \mathbf{r}=(\bar{x}, y, z)$ & $m_{b} \mathbf{r}=(x, \bar{y}+, z+)$ & $m_{c} \mathbf{r}=(x, y, \bar{z}+)$ & $m_{b} \mathbf{r}=(x+, \bar{y}+, z+)$ \\
\hline
\end{tabular}

the origin and $m_{c}$, a glide operation which takes $c$ into $-c$ followed by a translation through $(b / 2) \hat{\mathbf{b}}$. Thus the critical eigenvector (which is the spatial Fourier transform of the spin distribution) must not only be an eigenvector of the inverse susceptibility matrix, but it must also simultaneously be an eigenvector of both $2_{a}$ and $m_{c}$. Since $\left[2_{a}\right]^{2}=1$, the eigenvalues of $2_{a}$ must be $\lambda\left(2_{a}\right) \equiv \lambda= \pm 1$. Since $\left[m_{c}\right]^{2}$ is a translation along the $b$ axis, the eigenvalues of $m_{c}$ must be $\lambda\left(m_{c}\right) \equiv \lambda^{\prime}= \pm \exp \left(i b q_{b} / 2\right)= \pm 1$. Thus, if we assume continuous transitions, there can only be four distinct symmetries of ordered phases, corresponding to independently selecting the eigenvalues of $2_{a}$ and $m_{c}$. The corresponding eigenvectors must be of the form

$$
\begin{aligned}
& S(q, 1)=\left(\alpha_{1}, \alpha_{2}, \alpha_{3}\right) \xi, \quad S(q, 2)=\lambda\left(\alpha_{1},-\alpha_{2},-\alpha_{3}\right) \xi, \\
& S(q, 3)=\lambda \lambda^{\prime}\left(-\alpha_{1}, \alpha_{2},-\alpha_{3}\right) \xi^{3}, \quad S(q, 4)=\lambda^{\prime}\left(-\alpha_{1},-\alpha_{2},+\alpha_{3}\right) / \xi^{3}, \\
& S(q, 5)=\left([1+\lambda] \alpha_{4},[1-\lambda] \alpha_{5},[1-\lambda] \alpha_{6}\right), \\
& S(q, 6)=-\lambda^{\prime}\left([1+\lambda] \alpha_{4},[1-\lambda] \alpha_{5},[1-\lambda] \alpha_{6}\right) \xi^{2},
\end{aligned}
$$

where $\xi=\exp \left(i q_{x} a / 4\right)$ and the $\alpha_{n}$ assume arbitrary complex values. To check this note that under $2_{a}$ sublattices \#1 and \#2 are interchanged as are \#3 and \#4, whereas under $m_{c}$ sublattices \#1 and \#4 are interchanged as are \#2 and \#3. Note that $2_{a}$ changes the signs of the $b$ and $c$-components of spin, while $m_{c}$ changes the signs of the $a$ and $b$ components of spin since spin is a pseudo-vector. This type of analysis, known as representation theory, is well-known and widely used. However, less well-known and often overlooked (as documented in [6]) is the fact that in these multiferroic systems the free energy must be invariant under the inversion symmetry $\mathcal{I}$ possessed by the lattice [7. One can then show [4, 13, 12, 6] that this symmetry fixes the phases of the $\alpha_{n}$ : for $\lambda=\lambda^{\prime}=1$, apart from an overall complex phase factor, $\alpha_{1}$ and $\alpha_{3}$ must be pure imaginary and $\alpha_{2}$ and $\alpha_{4}$ must be pure real. For other irreps [i. e. for the three other choices of the eigenvalues $\lambda\left(\mathcal{O}_{i}\right)$ ] one has analogous results. If (11) is generalized to include terms of fourth order in the spin variables, then a mean-field analysis for $T$ near $T_{c}$ shows that the overall amplitude of the spin wave function varies [proportionally to $\left.\left(T_{c}-T\right)^{1 / 2}\right]$, but the ratios among the $\alpha_{n}$ 's are nearly temperature independent. Therefore we replace $\alpha_{n}$ by $\sigma(\mathbf{q}) \alpha_{n}$ and require the normalization $\sum\left|\alpha_{n}\right|^{2}=1$. Thus the temperature dependence is incorporated in the order parameter $\sigma$. If we require that $\alpha_{4}$, say, be real, then the freedom to fix the overall phase is taken into account by allowing the order parameter to be complex, as one would expect, since the origin of the IC ordering is not fixed, at least within $F_{2}$. It should be noted that the order parameter inherits the symmetry of the spin functions, so that

$$
2_{a} \sigma=\lambda\left(2_{a}\right) \sigma=\lambda \sigma, \quad m_{c} \sigma=\lambda\left(m_{c}\right) \sigma=\lambda^{\prime} \sigma, \quad \mathcal{I} \sigma=\sigma^{*} .
$$


In the analysis of diffraction experiments one tries to fit the structure assuming in turn each of the four symmetries. In so doing one has not $3 n_{\tau}=18$ complex-valued fitting parameters, but rather the 4 or $5 \alpha$ 's of (3) (depending on which symmetry one is considering). However, the use of inversion further reduces the number of fitting parameters by half since their phases are fixed 12 .

Figure 2. Dielectric and magnetic phase diagrams of NVO (left, from [11, 4, 12]), $\mathrm{TbMnO}_{3}$ (center, from [14, 5]), and RFMO (right, from 17, 18]). In the dielectric phase diagram the direction of the spontaneous polarization (if any) is indicated. For NVO $T_{C} \approx 4 \mathrm{~K}, T_{<} \approx 6 \mathrm{~K}$, and $T_{>} \approx 9 \mathrm{~K}$. In the magnetic phase diagrams $\mathrm{P}$ denotes paramagnetic, HTI denotes a dominantly collinear IC phase with a single OP, LTI is a dominantly elliptically polarized phase with two OP's, and IC-TRI denotes the IC stacking of triangular lattice antiferromagnets.

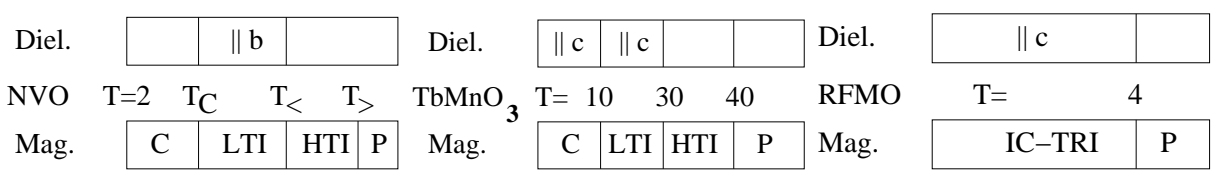

The left phase diagram in figure 2 shows the experimentally observed sequence of magnetic phases of NVO. As $T$ is lowered, the first ordered phase to appear (at $T_{>} \approx 9 \mathrm{~K}$ ) is the HTI phase, which has a single eigenvector associated with predominantly collinear sinusoidally modulated order. Analysis of experimental data indicates that the eigenvalues of this phase are 12 $\lambda\left(2_{x}\right)=-1$ and $\lambda\left(m_{c}\right)=+1$. At a lower temperature $\left(T_{<} \approx 6 \mathrm{~K}\right)$ the LTI phase appears with an additional order parameter associated with dominantly transverse spin order and with $\lambda\left(2_{x}\right)=+1$ and $\lambda\left(m_{c}\right)=+1$. The magnetic free energy which describes the development of these two successive ordering transitions is of the form $12,6,21$,

$$
F_{M}=a\left(T-T_{>}\right)\left|\sigma_{\mathrm{HTI}}\right|^{2}+b\left(T-T_{<}\right)\left|\sigma_{\mathrm{LTI}}\right|^{2}+\mathcal{O}\left(\sigma^{4}\right),
$$

where $a$ and $b$ are constants and $T_{>}$and $T_{<}$are the respective temperatures (when nonquadratic terms are ignored) at which $\sigma_{\text {HTI }}$ and $\sigma_{\text {LTI }}$ become nonzero. The unwritten terms in $F_{M}$, which are quartic in $\sigma$, favor fixed spin length. Thus $\sigma_{\mathrm{LTI}}$ is out of phase relative to $\sigma_{\mathrm{HTI}}$ and the spins thereby order in a spiral structure [12, 15, 16].

\section{2. $\mathrm{TbMnO} \mathrm{O}_{3}$}

The case of $\mathrm{TbMnO}_{3}$ is almost identical to that for NVO. Here the IC wave vector is of the form $(0, q, 0)[19,[5]$, so that the symmetry operations which leave it invariant are generated by the glide $m_{a}$ and the mirror $m_{c}$. The eigenvalues of $m_{c}\left(m_{a}\right)$ are \pm 1 $( \pm \Lambda)$, where $\Lambda=\exp (i \pi q)$. For $\lambda\left(m_{c}\right)=1$ and $\lambda\left(m_{a}\right)=\Lambda$ one has

$$
\begin{aligned}
& S(q, 1)=\alpha_{1} \hat{i}-\alpha_{2} \hat{j}-\alpha_{3} \hat{k}, \quad S(q, 2)=\alpha_{1} \hat{i}+\alpha_{2} \hat{j}+\alpha_{3} \hat{k}, \\
& S(q, 3)=-\alpha_{1} \hat{i}+\alpha_{2} \hat{j}-\alpha_{3} \hat{k}, \quad S(q, 4)=-\alpha_{1} \hat{i}-\alpha_{2} \hat{j}+\alpha_{3} \hat{k}, \\
& S(q, 5)=\alpha_{4} \hat{k}, \quad S(q, 6)=-\alpha_{5} \hat{k}, \\
& S(q, 7)=\alpha_{5} \hat{k}, \quad S(q, 8)=-\alpha_{4} \hat{k},
\end{aligned}
$$

where the $\alpha_{n}$ are arbitrary complex numbers. As for NVO one can now require that $F_{2}$ be invariant under $\mathcal{I}$. In this case the result is that apart from an overall phase factor, $\alpha_{n}$ for $n=1,2,3$ are real, $\alpha_{4}$ is an arbitrary complex number, and $\alpha_{5}=\alpha_{4}^{*}[5,13,6$. 
When inversion symmetry relates sites within the same Wyckoff orbit of the operators of the group of the wave vector (as it does for NVO), the complex phases get fixed, whereas when inversion relates sites in different Wyckoff orbits (as for the Tb sites here), the previously independent amplitudes of the two orbits are now related. Again, we replace $\alpha_{n}$ by $\sigma(\mathbf{q}) \alpha_{n}$, so that the temperature dependence of the spin function is essentially contained in the order parameter $\sigma(\mathbf{q})$ and the complex phase of the IC ordering is incorporated in the arbitrary complex phase of $\sigma(\mathbf{q})$ which transforms as

$$
m_{a} \sigma(\mathbf{q})=\lambda\left(m_{a}\right) \sigma(\mathbf{q}), \quad m_{c} \sigma(\mathbf{q})=\lambda\left(m_{c}\right) \sigma(\mathbf{q}), \quad \mathcal{I} \sigma(\mathbf{q})=\sigma(\mathbf{q})^{*} .
$$

The center phase diagram in figure 2 shows the sequence of magnetic phases of $\mathrm{TbMnO}_{3}$. As the temperature is lowered (through $T_{>}=40 \mathrm{~K}$ ) the first ordered phase to appear is the HTI phase in which the single eigenvector associated with predominantly collinear order appears with $\lambda\left(m_{c}\right)=-\exp (i \pi q) \equiv-\Lambda$ and $\lambda\left(m_{a}\right)=1$. At a lower temperature $\left(T_{<} \approx 30 \mathrm{~K}\right)$ the LTI phase appears with an additional order parameter associated with transverse spin order and with $\lambda\left(m_{c}\right)=-\Lambda$ and $\lambda\left(m_{a}\right)=-1$. The phenomenology of the magnetic phase diagram of $\mathrm{TbMnO}_{3}$ is very similar to that of NVO.

\section{3. $R F M O$}

The magnetic $\mathrm{Fe}$ ions in RFMO form triangular lattice planes which are stacked directly over one another, as shown in figure 1(c) 17. Below $T=180 \mathrm{~K}$ but above the magnetic ordering temperature $\left(\right.$ at $T_{c}=4 \mathrm{~K}$ ) the lattice has $\mathrm{P} \overline{3}$ symmetry 20 , so that the only symmetry operation (apart from $\mathcal{I}$ ) is a three-fold rotation $\mathcal{R}$ about the $c$-axis, which is perpendicular to the triangular lattice plane. At low fields, the spins within a single triangular lattice plane form a $120^{\circ}$ structure and as one moves from one plane to the next the spins are all rotated through an angle $\delta \phi=q_{c} c$, so that the component of the IC wave vector along $\hat{\mathbf{c}}$ is $q_{c}$ [18. To generate the $120^{\circ}$ structure, the in-plane component of the wave vector must be chosen to be at the corner, $\mathbf{X}$, of the Brillouin zone of the triangular lattice, i.e. $\mathbf{q}=\mathbf{X}+q_{c} \hat{\mathbf{c}}$. Then the symmetry operations $\mathcal{O}_{i}$ which leave the wave vector invariant are $\mathcal{R}$ and $\mathcal{R}^{-1}$. ( $\mathcal{R}$ takes $\mathbf{X}$ into a vector equivalent to $\mathbf{X}$.) We thus end up with a one-dimensional irrep $\Gamma$ and its complex conjugate $\Gamma^{*}$. The spin distribution is given by $[18,6$,

$$
\mathbf{S}(\mathbf{r})=\left[\sigma_{1}\left(q_{z}\right)(\hat{i}+i \hat{j})+\sigma_{2}\left(q_{z}\right)(\hat{i}-i \hat{j})\right] e^{i \mathbf{q} \cdot \mathbf{r}}+\text { c. c. },
$$

where $\mu=\exp (2 \pi i / 3)$. The order parameters transform as

$$
\mathcal{R} \boldsymbol{\sigma}_{n}\left(q_{z}\right)=\mu^{n} \boldsymbol{\sigma}_{n}\left(q_{z}\right), \quad \mathcal{I} \boldsymbol{\sigma}_{n}\left(q_{z}\right)=\boldsymbol{\sigma}_{3-n}\left(q_{z}\right)^{*} .
$$

The magnetic free energy up to order $\sigma^{4}$ is

$$
F=\left(T-T_{c}\right) \boldsymbol{\sigma}^{2}+u \boldsymbol{\sigma}^{4}+v\left|\sigma_{1}\left(q_{z}\right) \sigma_{2}\left(q_{z}\right)\right|^{2},
$$

where $\boldsymbol{\sigma}^{2} \equiv\left|\sigma_{1}\left(q_{z}\right)\right|^{2}+\left|\sigma_{2}\left(q_{z}\right)\right|^{2}$, and $u$ and $v$ are constants (with $u$ positive). It is found [18] that only one of the two order parameters is nonzero in a single domain, from which we deduce that $v$ must be positive. (This conclusion is confirmed by the appearance of ferroelectricity, as we will see in a moment.)

\subsection{Magnetoelectric Interaction}

Here we describe the ME interaction which leads to a spontaneous polarization induced by magnetic ordering which breaks inversion symmetry. For this purpose we show the dielectric phase diagrams of the three systems under consideration in figure 2. 
We write the free energy as

$$
F=F_{M}+F_{E}+V_{\text {int }},
$$

where $F_{M}\left(F_{E}\right)$ is the magnetic (dielectric) free energy and $V_{\text {int }}$ is the ME interaction which is responsible for the magnetically induced ferroelectricity.

We first consider $\mathrm{NVO}\left[4,12\right.$ and $\mathrm{TbMnO}_{3}$,5, 6]. Both have two magnetic ordered phases, the high-temperature incommensurate (HTI) phase at higher temperature $\left(T_{>}>T>T_{<}\right)$, described by a single order parameter $\sigma_{\mathrm{HTI}}$ for which spins are predominantly confined to the easiest direction, and the low-temperature incommensurate (LTI) phase (for $T<T_{<}$) in which a new order parameter $\sigma_{\text {LTI }}$ appears, describing ordering transverse to that of $\sigma_{\mathrm{HTI}}$. The order parameters are out of phase (to minimize the fourth order terms in the magnetic free energy) 12, and thus give rise to a magnetic spiral. These order parameters transform as specified by (41) and (7), respectively.

We have $F_{E}=(1 / 2) \chi_{E}^{-1} \mathbf{P}^{2}$, where $\chi_{E}$ is the dielectric susceptibility and $\mathbf{P}$ is the polarization vector. Because there is no tendency for ferroelectricity to form in the absence of magnetic ordering, $\chi_{E}$ never gets large. In the absence of ME coupling, the equilibrium value of $\mathbf{P}$ is zero. The $\mathrm{ME}$ interaction has to conserve wave vector and be invariant under time reversal. At lowest (quadratic) order in $\sigma$, it therefore must be of the form $V_{\text {int }} \sim \sigma(q) \sigma(-q) P \equiv \sigma \sigma^{*} P$. In the present situation, the two $\sigma$ 's can not both be HTI or LTI, because then $V_{\text {int }}$ would not be invariant under spatial inversion. So

$$
V_{\mathrm{int}}=\sum_{\gamma}\left[c_{\gamma} \sigma_{\mathrm{HTI}}(q) \sigma_{\mathrm{LTI}}(q)^{*}+c_{\gamma}^{*} \sigma_{\mathrm{HTI}}(q)^{*} \sigma_{\mathrm{LTI}}(q)\right] P_{\gamma},
$$

and to be invariant under inversion we must have $c_{\gamma}=i r_{\gamma}$, where $r_{\gamma}$ is real, so that [4]

$$
\begin{aligned}
V_{\text {int }} & =i \sum_{\gamma} r_{\gamma}\left[\sigma_{\text {HTI }}(q) \sigma_{\text {LTI }}(q)^{*}-\sigma_{\text {HTI }}(q)^{*} \sigma_{\text {LTI }}(q)\right] P_{\gamma} \\
& =2 \sin \left(\phi_{\text {HTI }}-\phi_{\text {LTI }}\right)\left|\sigma_{\text {HTI }} \sigma_{\text {LTI }}\right| \sum_{\gamma} r_{\gamma} P_{\gamma},
\end{aligned}
$$

where $\boldsymbol{\sigma}_{\mathrm{HTI}}=\left|\boldsymbol{\sigma}_{\mathrm{HTI}}\right| \exp \left(i \phi_{\mathrm{HTI}}\right)$ and similarly for $\boldsymbol{\sigma}_{\mathrm{LTI}}$. The transformation properties given in (4) and (77) for the order parameters under the mirror and glide operations then imply that $r_{\gamma}$ in (13) is only nonzero for $\gamma=b$ for NVO 4, 12, 13, 6] and $\gamma=c$ for $\mathrm{TbMnO}_{3}[5,13,6]$. The fact that $P$ is proportional to $\left|\sigma_{\mathrm{HTI}} \sigma_{\mathrm{LTI}}\right|$ has been experimentally verified for $\mathrm{NVO} 22$.

For RFMO the argument is slightly different. There (9) indicates that $\sigma_{1}\left(q_{z}\right) \sigma_{2}\left(q_{z}\right)^{*}$ is invariant under inversion (which changes the sign of $\mathbf{P}$ ). Thus (9) implies that the ME interaction quadratic in $\sigma$, which conserves wave vector, is [18, 6]

$$
V_{\text {int }}=\sum_{\gamma} r_{\gamma}\left[\left|\sigma_{1}\left(q_{z}\right)\right|^{2}-\left|\sigma_{2}\left(q_{z}\right)\right|^{2}\right] P_{\gamma}
$$

where $r_{\gamma}$ is real valued. Since the square bracket is invariant under the three-fold rotation $\mathcal{R}, P_{\gamma}$ must also be invariant under $\mathcal{R}$. So at this order $r_{\gamma}$ can only be nonzero for $\gamma=c$, as is observed [18]. At higher order 23] a transverse polarization is in principle possible. Note that $\mathcal{R}\left(P_{x}-i P_{y}\right)=\mu\left(P_{x}-i P_{y}\right)$ and $\mathcal{R} \sigma_{1} \sigma_{2}^{*}=\mu^{2} \sigma_{1} \sigma_{2}^{*}$. Then one can have an ME interaction of the form

$$
V_{\text {int }}^{(4)}=c\left[\left|\sigma_{1}\left(q_{z}\right)\right|^{2}-\left|\sigma_{2}\left(q_{z}\right)\right|^{2}\right] \sigma_{1}\left(q_{z}\right) \sigma_{2}\left(q_{z}\right)^{*}\left(P_{x}-i P_{y}\right)+\text { c.c. . }
$$


However, the fourth order terms written in (10) select $\left|\sigma_{1}\left(q_{z}\right)\right|=\left|\sigma_{2}\left(q_{z}\right)\right|$ if $v$ is negative and $\sigma_{1}\left(q_{z}\right) \sigma_{2}\left(q_{z}\right)=0$ if $v$ is positive. In either case $V_{\text {int }}^{(4)}$ does not come into play. Since the ordered phase is ferroelectric, we deduce that $v$ is positive and that only $P_{c}$ is nonzero. Then, within mean field theory, $P_{c}$ is proportional to $\left\langle|\sigma|^{2}\right\rangle$, as is the intensity of the magnetic Bragg peaks. This is experimentally confirmed [18. [

\section{125's}

Figure 3. (Color online) (a) Two views of the lattice structure of the 125's. (b) Symmetry operations of space group Pbam. $m_{\alpha}$ denotes a mirror or glide operation, $2_{\alpha}$ is a two-fold rotation or screw operation and $r+\equiv r+1 / 2$.
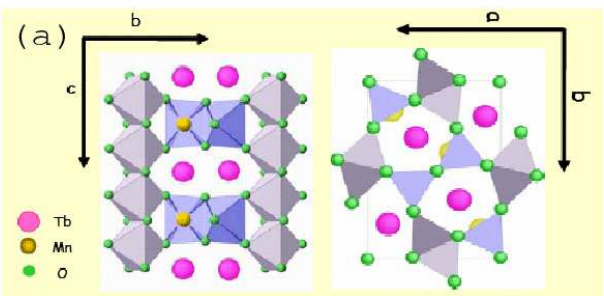

(b)

$$
\begin{array}{rlrl}
E \mathbf{r} & =(x, y, z) & 2_{\mathrm{a}} \mathbf{r} & =(x+, \bar{y}+, \bar{z}) \\
I \mathbf{r} & =(\bar{x}, \bar{y}, \bar{z}) & 2_{\mathrm{b}} \mathbf{r} & =(\bar{x}+, y+, \bar{z}) \\
2_{\mathrm{c}} \mathbf{r} & =(\bar{x}, \bar{y}, z) & \mathrm{m}_{\mathrm{a}} \mathbf{r}=(\bar{x}+, y+, z) \\
m_{\mathrm{c}} \mathbf{r} & =(x, y, \bar{z}) & \mathrm{m}_{\mathrm{b}} \mathbf{r}=(x+, \bar{y}+, z)
\end{array}
$$

We now consider the "125" orthorhombic (space group Pbam) family $\mathrm{RMn}_{2} \mathrm{O}_{5}$ (RMO), where $\mathrm{R}=\mathrm{Y}, \mathrm{Ho}, \mathrm{Er}, \mathrm{Dy}, \mathrm{Tb}$, Tm. Their lattice structure and the corresponding space group operations are shown in figure 3 . The paramagnetic unit cell of the RMO's contains 12 potentially magnetic ions: $4 \mathrm{Mn}^{3+}, 4 \mathrm{Mn}^{4+}$ and $4 \mathrm{R}^{3+}$. Experiments show that all the RMO's exhibit magnetic spin density wave ordering, with a wave vector $\mathbf{q}$ which undergoes a sequence of phase transitions 24]-29]. To discuss these phases we introduce the notation $\mathbf{q}=(U, 0, V)_{n}$, which we abbreviate as $(U, V)_{n}$ (in figure 5 these are denoted by $\left.U V_{n}\right)$. If $U=C(U=I)$, then $q_{x}=1 / 2$ $\left(q_{x}=1 / 2-\delta\right)$ and if $V=C(V=I)$, then $q_{z}=1 / 4\left(q_{z}=1 / 4+\epsilon\right)$, where the wave vector is in reciprocal lattice units and $\delta$ and $\epsilon$ are of order 0.01 and depend on temperature. $V=X$ includes the cases when $\epsilon \neq 0$ and when $\epsilon=0$. The subscript $n$, if it is given, indicates indicates the number ( 1 or 2 ) of OP's, see below. As the temperature $T$ decreases, all the RMO's (with the possible exception of $\mathrm{R}=\mathrm{Dy}$ ) first order below $T_{c}(\approx 45 \mathrm{~K})$, into an incommensurate $(I, I)$ phase with no ferroelectric (FE) order. For YMO (at $T_{F}=41 \mathrm{~K}$ ) 30, 31, ErMO (at $\left.T_{F}=39 \mathrm{~K}\right) 32$ and TmMO (at $T_{F}=39 \mathrm{~K}$ ) [33, this paraelectric incommensurate state gives way to an $(I, C)$ phase and this phase displays a weak FE moment $\mathbf{P}$ along the $b$-axis. Below $T_{C} \sim 37-39 \mathrm{~K}$, q locks into a commensurate $(\mathrm{CM})$ value $(C, C)$ and $P_{b}$ increases significantly [31]. TbMO 30, 36], HoMO 37, 38], and probably DyMO 37] go directly from the $(I, I)$ phase into the ferroelectric $(C, C)$ phase. At lower temperature (about 10-20K) most of the RMO's return to having some kind of incommensurate order $\llbracket$. We will not be concerned here with these low temperature phases, since their existence probably depends sensitively on the details of the spin-spin interactions. As we shall see, the behavior of the higher temperature phases can be described by a generic Landau free energy. The magneto-dielectric phase diagrams of various 125's are shown in figure 4

$\S$ However, critical fluctuations may imply different exponents for $P_{c}$ and $|\sigma|^{2}$, see Sec. 4 below.

|| This order may be commensurate but with a large unit cell. 
Figure 4. ME phase diagrams of ErMO 26, TmMO 28, YMO 34, 35, HoMO 32, 29, TbMO 27, and DyMO 39, 40, 38, 37. We do not indicate possible phase changes which have a dielectric signature but only a weak magnetic signature and hence may represent a minor spin reorientation. In Sec. 3.4 we argue that for $40<T<44 \mathrm{~K}$ DyMO is in an $(I, I)$ phase.

\begin{tabular}{|c|c|c|c|c|c|}
\hline Dielectric & \multicolumn{3}{|c|}{$\| \mathrm{b}$} & & \\
\hline ErMO & \multicolumn{2}{|c|}{$\mathrm{T}=11.5$} & 38 & \multicolumn{2}{|c|}{45} \\
\hline Magnetic & $(\mathrm{C}, 0, \mathrm{I})$ & $(\mathrm{C}, 0, \mathrm{C})$ & $(\mathrm{I}, 0, \mathrm{C})$ & $(\mathrm{I}, 0, \mathrm{I})$ & $\mathrm{P}$ \\
\hline Dielectric & \multicolumn{3}{|c|}{$\| \mathrm{b}$} & & \\
\hline YMO & \multicolumn{2}{|c|}{$\mathrm{T}=19$} & 38 & 41 & 45 \\
\hline Magnetic & LT & $(\mathrm{C}, 0, \mathrm{C})$ & $(\mathrm{I}, 0, \mathrm{C})$ & $(\mathrm{I}, 0, \mathrm{I})$ & $\mathrm{P}$ \\
\hline Dielectric & \multicolumn{3}{|c|}{$\| \mathrm{b}$} & & \\
\hline TbMO & \multicolumn{2}{|c|}{$\mathrm{T}=22$} & 7 & \multicolumn{2}{|c|}{42} \\
\hline Magnetic & LT & $(\mathrm{C}, 0, \mathrm{C})$ & $\mathrm{CM}+\mathrm{IC}$ & $(\mathrm{I}, 0, \mathrm{I})$ & $\mathrm{P}$ \\
\hline
\end{tabular}

\begin{tabular}{|c|c|c|c|c|c|}
\hline Dielectric & $? ?$ & $\|$ & & & \\
\hline TmMO & $\mathrm{T}=5$ & 25 & & 9 & \\
\hline Magnetic & \begin{tabular}{l|l} 
LTCM & LTIC
\end{tabular} & $(\mathrm{C}, 0, \mathrm{C})$ & $(\mathrm{I}, 0, \mathrm{C})$ & $(\mathrm{I}, 0, \mathrm{I})$ & $\mathrm{P}$ \\
\hline Dielectric & || b?? & $\| \mathrm{b}$ & & & \\
\hline HoМO & \multicolumn{3}{|c|}{$\mathrm{T}=20$} & \multicolumn{2}{|c|}{45} \\
\hline Magnetic & $(\mathrm{I}, 0, \mathrm{I})+\mathrm{CM}$ & \multicolumn{2}{|l|}{$(\mathrm{C}, 0, \mathrm{C})$} & $(\mathrm{I}, 0, \mathrm{I})$ & $\mathrm{P}$ \\
\hline Dielectric & \multicolumn{3}{|c|}{$\| \mathrm{b}$} & & \\
\hline DyMO & \multicolumn{2}{|l|}{$\mathrm{T}=14$} & 40 & \multicolumn{2}{|c|}{44} \\
\hline Magnetic & $(\mathrm{I}, 0, \mathrm{I})$ & $0, \mathrm{C})$ & $? ?$ & $? ?$ & $\mathrm{P}$ \\
\hline
\end{tabular}

Based on the symmetry of the OP's we construct a Landau theory for the various RMO's, which yields a generic phase diagram, shown in figure [5 which is independent of the detailed microscopic interactions 21. Each RMO has particular coupling constants which determine the wave vector q. Varying these parameters, $J_{x}$ for $q_{x}$ and $J_{z}$ for $q_{z}$, changes the value of the optimal $\mathbf{q}$ at which magnetic ordering occurs. The rest of this section is devoted to an explanation of this phase diagram (including the definitions of the various phases) and to a discussion of its consequences. This analysis is particularly relevant for the RMO's, because the microscopic theory of their multiferroicity is somewhat controversial. Our theory provides a unified explanation for the various sequences of phase transitions of the magnetic wave vector, and explains why ferroelectricity does or does not occur in the various magnetic phases. It also explains the occurrence of two distinct spin structures from neutron diffraction studies of the CM phase 35, 25. This phenomenological theory suggests several new experiments and makes a number of predictions, which can be tested experimentally.

\subsection{Magnetic Structure of the $(I, I)_{1}$ and $(I, C)_{1}$ Phases of the 125's}

Given the experimental information, we now analyze the various phases in the order in which they arise upon cooling from the $\mathrm{P}$ phase. The first phase which is encountered is of the $(I, I)$ type. Since $q_{z}=1 / 4$ plays no special symmetry role, it is convenient to discuss the $(I, I)$ and the $(I, C)$ phases together. Here, the star of $\mathbf{q}$ consists of four wave vectors, namely, $\mathbf{q}_{ \pm}=( \pm(1 / 2-\delta), 0,1 / 4+\beta)$ and their negatives. Each wave vector is invariant under unity and $m_{y}$. This symmetry group has two one-dimensional (1D) irreps, $\Gamma_{a}$ and $\Gamma_{b}$, with complex OP's as amplitudes. By symmetry, all the wave vectors of the star must have degenerate eigenvalues of (1). Therefore, we introduce complex OP's, $\sigma_{a}^{+} \equiv \sigma_{a}\left(\mathbf{q}_{+}^{(a)}\right)$ and $\sigma_{a}^{-} \equiv \sigma_{a}\left(\mathbf{q}_{-}^{(a)}\right)$ associated with irrep $\Gamma_{a}$ at its wave vectors $\mathbf{q}_{+}^{(a)}$, and similarly for $\Gamma_{b}$. Here $\mathbf{q}_{+}^{(a)}$ and $\mathbf{q}_{-}^{(a)}\left(\mathbf{q}_{+}^{(b)}\right.$ and $\left.\mathbf{q}_{-}^{(b)}\right)$ are defined to be the wave vectors at which the $\left\langle\sigma_{a}(\mathbf{q}) \sigma_{a}(\mathbf{q})^{*}\right\rangle\left(\left\langle\sigma_{b}(\mathbf{q}) \sigma_{b}(\mathbf{q})^{*}\right\rangle\right)$ susceptibility is maximal as $T \rightarrow T_{c a}\left(T \rightarrow T_{c b}\right)$. Specific basis functions are given elsewhere [41], where it is also shown that they transform as

$m_{y} \sigma_{s}\left(\mathbf{q}_{ \pm}^{(s)}\right)=\lambda_{s} \sigma_{s}\left(\mathbf{q}_{ \pm}^{(s)}\right), \quad \mathcal{I}_{\sigma_{s}}\left(\mathbf{q}_{ \pm}\right)=\kappa_{ \pm} \sigma_{s}\left(\mathbf{q}_{ \pm}^{(s)}\right)^{*}, \quad 2_{c} \sigma_{s}\left(\mathbf{q}_{ \pm}^{(\mathbf{s})}\right)=\eta^{2} \sigma_{s}\left(-\mathbf{q}_{\mp}^{(s)}\right)$, 
Order Parameters and Phase Diagrams of Multiferroics

Figure 5. (Color) Left: Schematic 3D phase diagram for $\mathbf{q}$ near $(1 / 2,0,1 / 4)$. The top (red) surface represents the phase boundary between the $\mathrm{P}$ and $(I, I)_{1}$ phases (where both $q_{x}$ and $q_{z}$ are incommensurate). Below the blue surface, which is a parabola in $J_{z}$ (depending only weakly on $J_{x}$ ), one has $q_{x}=1 / 4$, in phases $(I, C)_{1}$ and $(I, C)_{2}$. The green surface represents $(I, I)_{1} \rightarrow(I, I)_{2}$ and $(I, C)_{1} \rightarrow(I, C)_{2}$ (the subscripts 1 and 2 denote the number of $1 \mathrm{D}$ irreps which order). Below the orange surface, which is a parabola in $J_{x}$ (depending only weakly on $\left.J_{z}\right)$, one has $q_{x}=1 / 2$. Right: A cut at constant $q_{x}$. The $(I, I)_{2}$ and $(I, C)_{2}$ phases disappear below the orange surface (as $\left.q_{x} \rightarrow 1 / 2\right)$, where one has a 2D irrep. The dashed and dotted lines are possible trajectories followed by specific RMO's as the temperature is varied.
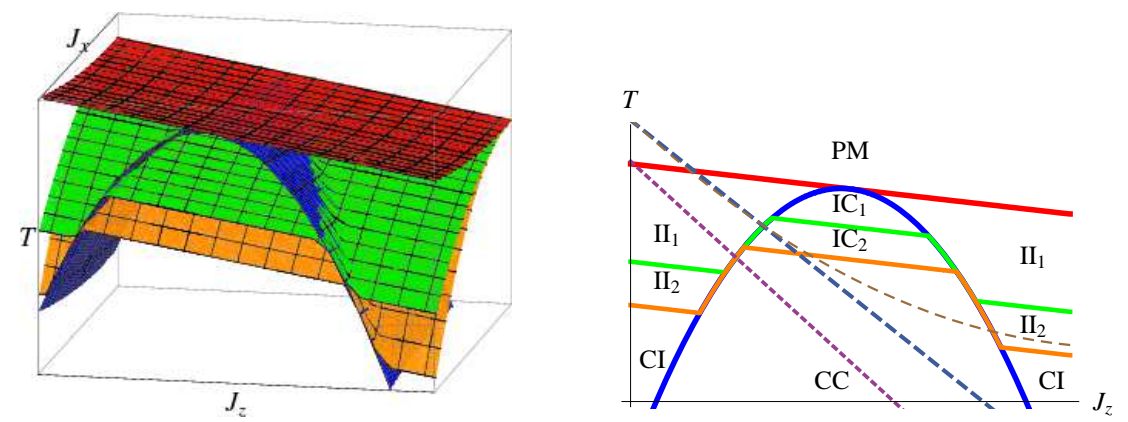

where $\lambda_{a}=-\lambda_{b}=\exp \left(i \pi q_{x}\right) \equiv \eta^{*}$ and $\kappa_{ \pm}=\eta^{2} \exp \left(\mp 2 \pi i q_{z}\right)$.

As one cools from the $\mathrm{P}$ phase, one must enter a phase described by a single irrep. Arbitrarily choosing this irrep as $\Gamma_{a}$, the corresponding free energy is

$$
\begin{aligned}
F^{(a)}= & \left(T-T_{c a}\right)\left[\left|\sigma_{a}^{+}\right|^{2}+\left|\sigma_{a}^{-}\right|^{2}\right]+c_{1}\left[\left|\sigma_{a}^{+}\right|^{2}+\left|\sigma_{a}^{-}\right|^{2}\right]^{2} \\
& +c_{2}\left|\sigma_{a}^{+} \sigma_{a}^{-}\right|^{2}+c_{3}\left[\left(\sigma_{a}^{+} \sigma_{a}^{-}\right)^{2}+\text { c.c. }\right] \delta_{4 q_{z}, 1},
\end{aligned}
$$

and analogously for $\Gamma_{b}$. The coefficients $c_{1}, c_{2}$ and $c_{3}$ may differ for $F^{(b)}$, and we assume that $T_{c b}<T_{c a}$. When $q_{z} \neq 1 / 4$, this free energy describes the $(I, I)_{1}$ phase (the subscript 1 indicates a single irrep). In this phase, we have $\left|\sigma_{a}^{+}\right|=\left|\sigma_{a}^{-}\right|$if $c_{2}<0$, while only one of $\sigma_{a}^{+}$or $\sigma_{a}^{-}$orders if $c_{2}>0$. Replacing $\left(T-T_{c a}\right)$ by $r\left(q_{0}\right)$, where $q_{0}$ is the wave vector which minimizes $r(q)$, the corresponding minimal free energies in the $(I, I)_{1}$ phase are given by $F_{I I}=-r\left(q_{0}\right)^{2} / w$, with $w=4 c_{1}\left(4 c_{1}+c_{2}\right)$ if $c_{2}>0\left(c_{2}<0\right)$.

If $q_{z}$ is close to $1 / 4$ then the last (Umklapp) term in (18) can lock $q_{z}$ to $1 / 4$, via a weakly first order transition. Clearly, this term arises only when both $\sigma_{a}^{+}$and $\sigma_{a}^{-}$ order, which would now happen only if $c_{2}-2\left|c_{3}\right|<0$. In this case, one again has $\left|\sigma_{a}^{+}\right|=\left|\sigma_{a}^{-}\right|$and $F_{I C}=-r(1 / 4)^{2} / w^{\prime}$, with $w^{\prime}=4 c_{1}+c_{2}-2\left|c_{3}\right|$. One would then have a first order transition from $(I, I)_{1}$ into $(I, C)_{1}$ when $F_{I I}=F_{I C}$. Since $r(q)$ has a minimum at $q_{0}$, we have $r(1 / 4) \approx r\left(q_{0}\right)+\alpha\left(1 / 4-q_{0}\right)^{2}$. Thus, the transition would occur when $r\left(q_{0}\right)+\alpha\left(1 / 4-q_{0}\right)^{2}=r\left(q_{0}\right)\left(w^{\prime} / w\right)^{1 / 2}$. Remembering that $r\left(q_{0}\right)=T-T_{c a}$, we have $q_{z}-1 / 4 \propto\left(T_{c a}-T\right)^{1 / 2}$. Furthermore, since $q_{z}=1 / 4$ is not a special point, we expect $q_{0}$ to be a linear function of $J_{z}$, hence $1 / 4-q_{0} \propto J_{z}-J_{z c}$, where $J_{z c}$ is the special value of $J_{z}$ associated with the transition from the $\mathrm{P}$ state into the state with $q_{z}=1 / 4$. Thus, the transition from $(I, I)_{1}$ into $(I, C)_{1}$ occurs at $T=T_{F}$, with

$$
T_{c a}-T_{F} \propto\left(J_{z}-J_{z c}\right)^{2},
$$

as shown in figure 6a. This parabolic relation is a mean-field result.

Now consider the implications of having $q_{z}$ locked to the value $1 / 4$ in the $(I, C)$ phases. From (18) we see that for this locking to occur, both wave vectors $\mathbf{q}_{+}$and $\mathbf{q}_{-}$ 
Figure 6. (Color online) Phase diagrams (a) for $q_{z} \approx 1 / 4$, based on (19) and (b) for $q_{x} \approx 1 / 2$, based on (31) and (32), when the (I,I) $)_{1}-(\mathrm{I}, \mathrm{I})_{2}$ phase boundary (dashed line) is preempted by locking $q_{x}$ to $q_{x}=1 / 2$. Parabolas shown as a function of $J_{x}\left(J_{z}\right)$ are weak functions of $J_{z}\left(J_{x}\right)$. (c) $r_{ \pm}\left(q_{x}\right)$ for nonzero $\Delta J_{x}$, based on (30). The OP associated with each point is given in the box along with the parameters which characterize the wave function, as explained in 41]. In (a) and (b) the points $\mathrm{M}$ and $\mathrm{M}$ ' are multicritical points that can only be reached by adjusting both the temperature and some additional control parameter.
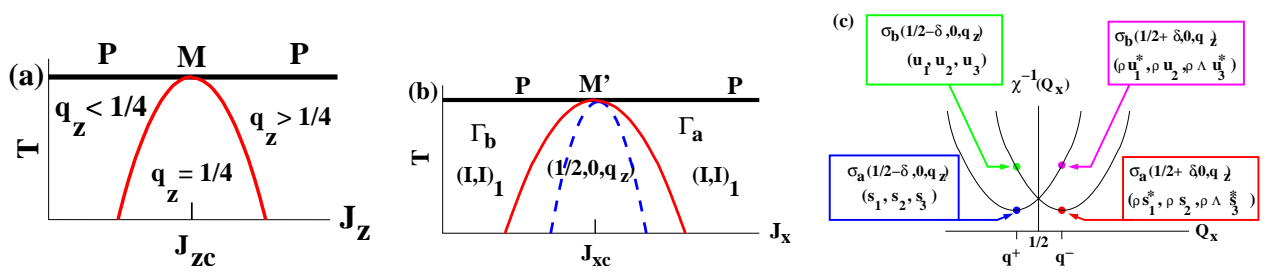

must appear (in the same domain). Then, since we do not allow a direct transition from the $\mathrm{P}$ phase to the $(I, C)_{1}$ phase (we ignore the unlikely case of a multicritical point, at which $J_{z}=J_{z c}$ ), the question is whether or not a single domain of the neighboring $(I, I)_{1}$ phase has two wave vectors. From (18), the condition to have two wave vectors is that $c_{2}<0$. An alternate scenario would be that $c_{2}>0$ and the two wave vectors do not order simultaneously (in the same domain) in the $(I, I)_{1}$ phase. In that case, barring the existence of an as-yet-undetected phase boundary, the two wave vectors would have to appear in conjunction with the phase transition between the $(I, I)_{1}$ and $(I, C)_{1}$ phases. For the two wave vectors not to be present in the $(I, I)_{1}$ phase would imply that $c_{2}>0$. Then if $c_{2}-2\left|c_{3}\right|<0$, the two wave vectors would appear at the $(I, I)_{1} \rightarrow(I, C)_{1}$ phase transition. It would be interesting to experimentally determine (following the logic of Ref. [42]) which scenario actually occurs, i. e. whether or not the $(I, I)_{1}$ phase has simultaneous condensation at both wave vectors. For this purpose, it would be interesting to perform an experiment analogous to that of Ref. 42]. Here, since the ME interaction is present, one could use an electric field parallel to one of the wave vectors to manipulate the domains.

\subsection{Magnetoelectric Structure of the $(I, I)_{2}$ and $(I, C)_{2}$ Phases of the 125's}

Similarly to NVO and $\mathrm{TbMnO}_{3}$, the second 1D irrep $\Gamma_{b}$ may order upon further cooling. In addition to the 'decoupled' free energies $F^{(a)}$ and $F^{(b)}$, the total free energy now contains many terms which couple the OP's $\sigma_{a}^{ \pm}$and $\sigma_{b}^{ \pm}$. We start by discussing the $(I, I)_{2}$ phase, where all the wave vector components remain incommensurate. Then the quartic terms which couple the two sets of OP's are given by

$$
F_{4}^{(x)}=c_{4}\left[\left|\sigma_{a}^{+} \sigma_{b}^{+}\right|^{2}+\left|\sigma_{a}^{-} \sigma_{b}^{-}\right|^{2}\right]+c_{5}\left[\left|\sigma_{a}^{+} \sigma_{b}^{-}\right|^{2}+\left|\sigma_{a}^{-} \sigma_{b}^{+}\right|^{2}\right]+\Delta,
$$

where $\Delta$ contains the locking terms,

$$
\begin{aligned}
\Delta= & c_{6}\left[\left(\sigma_{a}^{+} \sigma_{b}^{+*}\right)^{2}+\left(\sigma_{a}^{-} \sigma_{b}^{-*}\right)^{2}+\left(\sigma_{b}^{+} \sigma_{a}^{+*}\right)^{2}+\left(\sigma_{b}^{-} \sigma_{a}^{-*}\right)^{2}\right] \delta_{\mathbf{q}_{+}^{(a)}, \mathbf{q}_{+}^{(b)}} \\
& +c_{7}\left[\sigma_{a}^{+} \sigma_{a}^{-}\left(\sigma_{b}^{+} \sigma_{b}^{-}\right)^{*}+\left(\sigma_{a}^{+} \sigma_{a}^{-}\right)^{*} \sigma_{b}^{+} \sigma_{b}^{-}\right] \delta_{q_{+, z}^{(a)}, q_{+, z}^{(b)}} \\
& +c_{8}\left[\sigma_{a}^{+} \sigma_{b}^{-}\left(\sigma_{b}^{+} \sigma_{a}^{-}\right)^{*}+\left(\sigma_{a}^{+} \sigma_{b}^{-}\right)^{*} \sigma_{b}^{+} \sigma_{a}^{-}\right] \delta_{q_{+, x}^{(a)}, q_{+, x}^{(b)}}
\end{aligned}
$$

Notice that so far we have not assumed that $\mathbf{q}_{ \pm}^{(a)}$ and $\mathbf{q}_{ \pm}^{(b)}$ are identical. If the exchange interactions were isotropic, then the inverse susceptibility would be invariant 
under a global rotation of all spin directions. Here, in the generic case, we have small anisotropic interactions which break this degeneracy and, in principle, would cause these critical wave vectors to be slightly different. In this case, the quartic terms $\Delta$ can lock the wave vectors of the two modes into equality, as happens for NVO 12 . The mechanism for this locking is as follows. Assume that, say, $\sigma_{a}$ orders first at $T=T_{c a}$, and for simplicity we first treat the case with only a single wave vector, so that, say, $\left\langle\sigma_{a}^{+}\right\rangle$, but not $\left\langle\sigma_{a}^{-}\right\rangle$, is nonzero. In analogy with what happens for $\mathrm{NVO}$ [12, we assume that $\mathbf{q}_{+}^{(a)}$ is almost equal to $\mathbf{q}_{+}^{(b)}$, at which the inverse susceptibility $\chi_{b}^{-1}(\mathbf{q})$ of $\sigma_{b}$ has its minimum. For $T_{c a}>T>T_{c b}$ this minimum in $\chi_{b}^{-1}(\mathbf{q})$ is positive since $\sigma_{b}$ has not yet ordered. Now, the quartic terms $\Delta$ give rise to an effective quadratic term, $V_{2, \text { eff }}$. Since only $\left\langle\sigma_{a}^{+}\right\rangle$is nonzero, we have

$$
V_{2, \text { eff }}=c_{6}\left[\left\langle\sigma_{a}^{+}\right\rangle^{2}\left(\sigma_{b}^{+*}\right)^{2}+\left(\sigma_{b}^{+}\right)^{2}\left\langle\sigma_{a}^{+*}\right\rangle^{2}\right] \delta_{\mathbf{q}_{+}^{(a)}, \mathbf{q}_{+}^{(b)}},
$$

where $\langle X\rangle$ indicates the thermal average of $X$. Even before $\sigma_{b}^{+}$orders, this term gives an additional contribution [beyond $\left(T-T_{c b}\right)$ ] to the inverse susceptibility of $\sigma_{b}^{+}$, but only when $\mathbf{q}_{+}^{(a)}=\mathbf{q}_{+}^{(b)}$. Since this additional term depends on the relative phase of the $\sigma_{a}$ 's and the $\sigma_{b}$ 's, the minimization of this term fixes the phase of $\sigma_{b}^{+}$, reducing its symmetry from that of the XY model (two components of a complex number) to that of an Ising model. The minimzation always leads to a negative contribution to the inverse susceptibility of $\sigma_{b}^{+}$. If $\left|\left\langle\sigma_{a}^{+}\right\rangle\right|^{2}$ is sufficiently large, this term can thereby shift the minimum in the $\sigma_{b}^{+}$inverse susceptibility from the wave vector $\mathbf{q}_{+}^{(b)}$ (which it would have had when $\Delta=0$ ) into equality with $\mathbf{q}_{+}^{(a)}$. Also, the star of the wave vector associated with $\sigma_{b}^{+}$now contains only the two vectors $\mathbf{q}_{+}^{(a)}$ and $-\mathbf{q}_{+}^{(a)}$. This scenario applies if the wave vectors for $\sigma_{a}$ and $\sigma_{b}$ are close enough to be locked to $\mathbf{q}_{+}^{(a)}$ by the term $V_{2 \text {,eff }}$ before reaching the temperature $T_{c b}$ at which $\sigma_{b}$ condenses. This, in turn, relies on the smallness of the anisotropic terms which cause $\mathbf{q}_{+}^{(a)}$ to differ from $\mathbf{q}_{+}^{(b)}$.

If both $\sigma_{a}\left(\mathbf{q}_{+}^{(a)}\right)$ and $\sigma_{a}\left(\mathbf{q}_{-}^{(a)}\right)$ condense at $T=T_{c a}$, then we need to consider all the terms in (21). In the $(I, I)_{1}$ phase, both $\left\langle\sigma_{a}^{+}\right\rangle=x e^{i \phi}$ and $\left\langle\sigma_{a}^{-}\right\rangle=x e^{i \chi}$ break the symmetry and have well defined phases $\phi$ and $\chi$ ( $x$ is a real number). Substituting these values into (21) the yields a quadratic form in the four real and imaginary parts of $e^{i \phi} \sigma_{b}^{+}$and $e^{i \chi} \sigma_{b}^{-}$, with eigenvalues $2 x^{2}\left[c_{6} \pm\left(c_{7}+c_{8}\right)\right]$ and $2 x^{2}\left[c_{6} \pm\left(c_{7}-c_{8}\right)\right]$. Since only one of these eigenvalues is lowest, only one combination of the four OP components of $\sigma_{b}^{ \pm}$orders, and thus we still have an Ising-like ordering into $(I, C)_{2}$. In any case, we henceforth assume that both OP's have the same critical wave vectors.

Experimentally, it seems that the phase $(I, I)_{2}$ has never been observed. Instead, the phase with two OP's below $(I, I)_{1}$ seems to be of the $(I, C)$ kind. Therefore, we now consider the possible locking of $q_{z}$ to $1 / 4$, which would correspond to the appearance of the $(I, C)_{2}$ phase. When $q_{z}$ is close to $1 / 4$, (20) must include additional Umklapp terms, which are also consistent with the symmetry of (17) and which lock $q_{z}$ to $1 / 4$. For $q_{x} \neq 1 / 2$, these are

$$
\left.U_{a b}=\left\{c_{9} \sigma_{a}^{+} \sigma_{b}^{+} \sigma_{a}^{-} \sigma_{b}^{-}+c_{10}\left[\left(\sigma_{a}^{+} \sigma_{b}^{-}\right)^{2}+\sigma_{a}^{-} \sigma_{b}^{+}\right)^{2}\right]+\text { c.c. }\right\} \delta_{4 q_{z}, 1},
$$

where $c_{9}$ and $c_{10}$ are real. The locking is stronger when two irreps, rather than a single irrep as in (18), are present, because then the additional terms of (23) come into play. However, in either case, note that this locking requires the presence of both wave vectors $\mathbf{q}_{+}$and $\mathbf{q}_{-}$.

Finally, we discuss the ME interactions in the $(I, I)$ and $(I, C)$ phases. In analogy with (13), the lowest order ME interaction which is invariant under the operations of 
(17) is

$$
V_{\mathrm{int}}=\operatorname{ir} P_{y} \sum_{ \pm}\left[\sigma_{a}\left(\mathbf{q}_{ \pm}\right) \sigma_{b}\left(\mathbf{q}_{ \pm}\right)^{*}-\sigma_{a}\left(\mathbf{q}_{ \pm}\right)^{*} \sigma_{b}\left(\mathbf{q}_{ \pm}\right)\right] .
$$

Thus, in the $(I, I)$ and in the $(I, C)$ phases, at this order, ferroelectricity requires the presence of two order parameters which are not in phase with one another. At fourth order in the magnetic order parameters, the ME interaction can lead to small spontaneous polarizations in the other coordinate directions, but due to space limitations we refer the reader to [21].

\subsection{Magnetoelectric Structure of the $(C, X)$ Phases}

This case includes both $X=I\left(q_{z}=1 / 4+\epsilon\right)$ and $X=C\left(q_{z}=1 / 4\right)$. Because $\mathbf{q}$ is on the Brillouin zone boundary $\left(q_{x}=1 / 2\right)$, the wave vector is invariant under $m_{a}$ and $m_{b}$, and the star of $\mathbf{q}$ consists of $\mathbf{q}$ and $-\mathbf{q}$. These operations lead to a two-dimensional irrep [43, 6] and we choose the basis functions as in table XVI of [6]. The actual wave function is a linear combination of the two basis functions with complex amplitudes $\sigma_{1}(\mathbf{q})$ and $\sigma_{2}(\mathbf{q})$. These are the OP's which characterize the magnetic structure and they transform as 6 ]

$$
m_{x} \sigma_{n}(\mathbf{q})=\zeta_{n} \sigma_{n}(\mathbf{q}), \quad m_{y} \sigma_{n}(\mathbf{q})=\zeta_{n} \sigma_{3-n}(\mathbf{q}), \quad \mathcal{I} \sigma_{n}(\mathbf{q})=\sigma_{3-n}(\mathbf{q})^{*},
$$

where $\zeta_{n} \equiv(-1)^{n+1}$. Consistent with these symmetries the magnetic free energy up to quartic order in $\sigma$ is

$$
\begin{aligned}
& F_{M}=\left(T-T_{C}\right)\left[\left|\sigma_{1}(\mathbf{q})\right|^{2}+\left|\sigma_{2}(\mathbf{q})\right|^{2}\right]+u\left[\left|\sigma_{1}(\mathbf{q})\right|^{2}+\left|\sigma_{2}(\mathbf{q})\right|^{2}\right]^{2} \\
& \quad+w\left|\sigma_{1}(\mathbf{q}) \sigma_{2}(\mathbf{q})\right|^{2}+v\left[\sigma_{1}(\mathbf{q}) \sigma_{2}(\mathbf{q})^{*}+\sigma_{2}(\mathbf{q}) \sigma_{1}(\mathbf{q})^{*}\right]^{2} \\
& \quad+\left[x\left(\sigma_{1}(\mathbf{q})^{4}+\sigma_{2}(\mathbf{q})^{4}\right)+y \sigma_{1}(\mathbf{q})^{2} \sigma_{2}(\mathbf{q})^{2}+\text { c. c. }\right] \delta_{4 q_{z}, 1},
\end{aligned}
$$

where $x$ and $y$ are real. Under the terms quadratic in $\sigma$ and those scaled by $u$, all directions in the four dimensional space of $\sigma_{1} \equiv \sigma_{1}(\mathbf{q})$ and $\sigma_{2} \equiv \sigma_{2}(\mathbf{q})$ are equally unstable relative to ordering. However, for $q_{z} \neq 1 / 4$, the fourth order terms select $\left|\sigma_{1}\right|=\left|\sigma_{2}\right|$ for $w+4 v<0$ if $v$ is negative, $\sigma_{1}= \pm i \sigma_{2}$ for $w<0$ if $v$ is positive, and $\sigma_{1} \sigma_{2}=0$ otherwise. For $q_{z}=1 / 4$ the terms in $x$ and $y$ are difficult to analyze analytically, but in many cases we find that the phases of $\sigma_{1}$ and of $\sigma_{2}$ can be chosen so that $F_{M}$ still has minima when either $\left|\sigma_{1}\right|=\left|\sigma_{2}\right|$ or $\sigma_{1} \sigma_{2}=0$.

Now we consider the dielectric properties. At quadratic order in $\sigma$, since $\mathcal{I} \sigma_{1} \sigma_{2}^{*}=\sigma_{1} \sigma_{2}^{*}$, (15) also applies to the 125's when $\mathbf{q}=\left(1 / 2,0, q_{z}\right)$ and then (25) indicates that $r_{\gamma}$ is only nonzero for $\gamma=b$. Including terms of higher order in $\sigma[23$. the ME interaction for the 125 's is of the form

$$
V_{\mathrm{int}}=r_{c}\left[\left|\sigma_{1}\right|^{2}-\left|\sigma_{2}\right|^{2}\right] P_{b}+i \sum_{\gamma} r_{\gamma}^{\prime}\left[\left|\sigma_{1}\right|^{2}-\left|\sigma_{2}\right|^{2}\right]\left[\sigma_{1} \sigma_{2}^{*}-\sigma_{1}^{*} \sigma_{2}\right] P_{\gamma},
$$

where, according to (25), the real coefficient $r_{\gamma}^{\prime}$ is only nonzero for $\gamma=a$. However, as mentioned above, (26) probably allows only either $\left|\sigma_{1}\right|=\left|\sigma_{2}\right|$ or $\sigma_{1} \sigma_{2}=0$, in which case the last term in (27) is inoperative. On the other hand, if $\sigma_{1} \sigma_{2}=0$ (so that, say, $\left.\sigma_{2}=0\right)$ and if one applies an electric field, $E_{a}$, in the a direction, which induces a nonzero value of $P_{a}$, the second term in (27) will induce a nonzero out-of-phase value in the order parameter, $\sigma_{2}$, that was zero for $E_{a}=0$. Then with $\left\langle\sigma_{1}\right\rangle \neq 0$, this effective linear coupling between $P_{a}$ and $\sigma_{2}$ gives rise to electromagnons [44, 45, 46].

The ME coupling can induce lattice displacements at wave vectors which are even integer multiples of the magnetic wave vector [47, 48. Since the results are 
particularly simple for the $(C, C)$ phase, where $\mathbf{q}=(1 / 2,0,1 / 4)$, we now discuss the lowest order interaction in that case. So far we considered a trilinear spin-phonon coupling involving $\sigma(\mathbf{q}) \sigma(\mathbf{q})^{*}$, which conserves wave vector and therefore couples to a uniform polarization. We now generalize this analysis, and consider terms of the form $\sigma(\mathbf{q})^{2}$ or $\sigma(\mathbf{q})^{* 2}$, which couple to phonon modes with wave vector $\pm 2 \mathbf{q}$. Within a reciprocal lattice vector, this phonon wave vector is equal to the antiferroelectric wave vector $(0,0,1 / 2)$. To construct this interaction we need the site symmetry analysis for this wave vector, which is the same as for the wave vector $(0,0,0)$ as given in Table I of [49]. There it is indicated that there are $15 \mathrm{~B}_{3 u}$ ( $x$-like) modes, $15 \mathrm{~B}_{2 u}$ ( $y$-like) modes, and $9 \mathrm{~B}_{1 u}$ ( $z$-like) phonon modes. An $x$-like mode, for instance, need not involve displacements along the $x$-axis; rather such a mode need only transform like $x$ under the space group operations. Accordingly, let $u_{\mathrm{AF}}(\gamma, \tau)$ denote such a phonon, where $\gamma$ labels the symmetry $(x, y$ or $z$, since we are only interested in vector-like modes which carry a polarization) and the index $\tau$ labels the occurrence. We use the transformation properties of (25) with $m_{z}=\mathcal{I} m_{x} m_{y}$, so that $m_{z} \sigma_{n}=\sigma_{n}^{*}$. Thus the combination $\left(\sigma_{1}^{2}+\sigma_{2}^{2}\right)$ is even under $m_{x}$ and $m_{y}$, so that the spin-phonon interaction contains the term

$$
V_{\mathrm{sp}-\mathrm{ph}, z}=\sum_{\tau}\left[i r_{\tau}\left(\sigma_{1}^{2}+\sigma_{2}^{2}\right)+\text { c.c. }\right] u_{\mathrm{AF}}(z, \tau),
$$

where $r_{\tau}$ is real, so that the square bracket is odd under $m_{z}$. Similarly $\sigma_{1} \sigma_{2}$ is odd under $m_{x}$ and $m_{y}$, so it cannot couple to a vector. Finally $\left(\sigma_{1}^{2}-\sigma_{2}^{2}\right)$ is even under $m_{x}$ and odd under $m_{y}$ and it gives rise to an ME interaction of the form

$$
V_{\mathrm{sp}-\mathrm{ph}, y}=\sum_{\tau}\left[r_{\tau}^{\prime}\left(\sigma_{1}^{2}-\sigma_{2}^{2}\right)+c . c .\right] u_{\mathrm{AF}}(y, \tau),
$$

where $r_{\tau}^{\prime}$ is real and we noted that the square bracket is even under $m_{z}$. In summary, at this order one can have antiferroelectricity with polarization along either $y$ or $z$.

We next analyze the tongue associated with $q_{x}=1 / 2$. Note that for a critical value, $J_{x c}$, of the control parameter $J_{x}$, the two branches [denoted $r_{ \pm}\left(q_{x}, J_{x}\right)$ ] of the quadratic coefficients $r\left(q_{x}\right)$ of the inverse susceptibility are degenerate and are minimal at $q_{x}=1 / 2$, so that $r_{ \pm}\left(q_{x}, J_{x c}\right)=r(0)+a\left(q_{x}-1 / 2\right)^{2}+\mathcal{O}\left(q_{x}-1 / 2\right)^{4}$, where $a$ is a positive constant. As $J_{x}$ is varied away from $J_{x c}$, a term in $r_{ \pm}\left(q_{x}\right)$ which is linear in $k_{x} \equiv\left(1 / 2-q_{x}\right)$ is allowed and generically is of order $\Delta J_{x} \equiv J_{x}-J_{x c} 41$. $\square$ The symmetry operation $m_{x}$ dictates that the spectrum of the two branches $r_{ \pm}\left(q_{x}\right)$ should be independent of the sign of $k_{x}$, as shown in figure $6 \mathrm{k}$, so that

$$
r_{ \pm}\left(k_{x}, J_{x}\right)=r(0)+a k_{x}^{2} \pm b k_{x}\left(J_{x}-J_{x c}\right) .
$$

and for concreteness we assume that the constant $b$ is negative and that $J_{x}>J_{x c}$. Symmetry thus implies the existence of equivalent minima at $k_{x}=\mp b\left(J_{x}-J_{x c}\right) /(2 a) \equiv$ $k^{ \pm}$. Thus at its minimum $r_{ \pm}\left(k_{x}\right)$ assumes the value $r\left(k_{x}=0\right)-\alpha^{\prime}\left(J_{x}-J_{x c}\right)^{2}$, where $\alpha^{\prime}$ is a constant. Accordingly, we can adopt the argument leading to (19), to the present case and obtain

$$
T_{c a}-T_{C} \propto\left(J_{x}-J_{x c}\right)^{2},
$$

9 As explained in [50, the largest polarization will come from $r_{\alpha}$-like modes, which have displacements in the $r_{\alpha}$-direction.

+ To see the existence of such a term consider the approximation in which, for a system with isotropic exchange interactions $J\left(\mathbf{r}, \mathbf{r}^{\prime}\right)$ between spins at $\mathbf{r}$ and $\mathbf{r}^{\prime}$, one has, for $\tau \neq \tau^{\prime}$ and $\mathbf{q}=\left(1 / 2-\delta, 0, q_{z}\right)$ that $\left.\left.\chi_{\tau, \alpha ; \tau^{\prime} \alpha}^{-1}(\mathbf{q})=J\left(\boldsymbol{\tau}, \mathbf{a}+\boldsymbol{\tau}^{\prime}\right) \exp [\pi i-2 \pi i \delta)\right]+J\left(\boldsymbol{\tau},-\mathbf{a}+\boldsymbol{\tau}^{\prime}\right) \exp [-\pi i+2 \pi i \delta)\right]$. As long as the sites do not sit at a center of inversion symmetry, these two terms will have different amplitudes and will give an imaginary contribution which is linear in both $\delta$ and $J$. 
where $T_{C}$ is the phase boundary between the $(I, X)$ and $(C, X)$ phases. (This phase boundary is the solid line in Fig. 6 b.)

The structure of (30) also allows us to discuss the phase boundary $T_{1 \rightarrow 2}$ between the $(I, I)_{1}$ and $(I, I)_{2}$ phases. For that purpose we compare (30) with (18) (and with its analog for $\left.F^{(b)}\right)$ and identify $r_{+}$with $T-T_{c a}$ and $r_{-}$with $T-T_{c b}$. We thereby find that

$$
T_{c, a}-T_{c, b}=2 b k^{+}\left(J_{x}-J_{x c}\right) \sim c\left(J_{x}-J_{x c}\right)^{2},
$$

where $c$ is a constant. Thus $T_{1 \rightarrow 2}$ is proportional to $\left(J_{x}-J_{x c}\right)^{2}$. Depending on the parameters, this parabolic tongue can be either narrower or wider than that considered above for locking $q_{x}$ to $q_{x}=1 / 2$. In the figure we show the former case, since the $(I, I)_{2}$ phase has not been observed for any of the 125 's.

\subsection{Generic Phase Diagram for $\mathrm{RMn}_{2} \mathrm{O}_{5}$}

We now explain how the generic phase diagram of figure 5 describes the various RMO's. Since $q_{z}=1 / 4$ is not a high symmetry point, we can not condense from the $\mathrm{P}$ phase into $q_{z}=1 / 4$ unless we adjust the $J$ 's appropriately to reach this higher order multicritical point. Since we reject this accidental possibility, the first ordered phase we encounter has $q_{z} \neq 1 / 4$. Although $q_{x}=1 / 2$ is a special value (characteristic of antiferromagnetically doubling the size of the unit cell), the result shown in figure 6b indicates that a continuous transition from the $\mathrm{P}$ phase into a $(C, I)$ phase is not allowed because it would also involve a multicritical point. For the RMO's (except $\mathrm{R}=$ Dy which we discuss separately), experiment shows that the first ordered phase is $(I, I)$ and this case is shown in figure 5. From now on we arbitrarily set $T_{c, a}>T_{c, b}$ (since we reject the possibility of accidental equality). Consequently we identify that the transition from the $\mathrm{P}$ phase is into an ordered phase $(I, I)_{1}$ with a single OP $\sigma_{a}$ (except for the star of $\mathbf{q}$ ). For a single OP, (24) provides a phenomenological explanation for why this phase is not ferroelectric. As discussed above, we assume that in the $(I, I)_{1}$ the phases $\mathbf{q}_{ \pm}^{(a)}$ and $\mathbf{q}_{ \pm}^{(b)}$ become locked into equality without crossing a phase boundary. For the phases with $q_{x} \neq 1 / 4$, experiments have not yet indicated whether the two wave vectors $\mathbf{q}_{ \pm}$occur in separate domains, or whether the true state is the superposition, within a single domain, of the two wave vectors. As $T$ is further reduced through the $(I, I)_{1}$ phase, a second continuous transition could occur, producing a phase $(I, I)_{2}$ in which both OP's $\sigma_{a}$ and $\sigma_{b}$ are nonzero (as in NVO [4, 6] or $\mathrm{TbMnO}_{3}[5]$ ).

The above description applies for $J_{z}$ relatively far away from $J_{z c}$, i.e. $q_{z}$ relatively far away from $1 / 4$. If $q_{z}=1 / 4$, one goes directly from the $\mathrm{P}$ phase into the $(I, C)_{1}$ phase, which is similar to the $(I, I)_{1}$ phase. Upon cooling, the OP related to the other 1D irrep tends to order, and one has a transition into the $(I, C)_{2}$ phase. This transition happens at a higher temperature than that for $(I, I)_{1} \rightarrow(I, I)_{2}$, due to Umklapp terms like (23), which enhance the tendency of $\sigma_{b}\left(q_{x}, 0, \pm 1 / 4\right)$ to order (compared to $\sigma\left(q_{x}, 0, q_{z}\right)$ with an IC $\left.q_{z}\right)$. If $q_{z}$ is close to $1 / 4$, one first goes from the $\mathrm{P}$ phase into the $(I, I)_{1}$ phase, but then the Umklapp terms cause a transition into the $(I, C)_{1}$ phase, and one ends up with the phase diagram shown on the RHS of figure 5.

As the temperature is lowered, each individual RMO follows some trajectory in the parameter space. The RHS plot in figure 5 shows possible projections of such trajectories. The trajectories, as well as the optimal wave vectors, are assumed to have some temperature dependence, which can originate from the elimination 
Table 2. Values of $q_{x}, q_{z}$, and $\Pi \equiv\left|1 / 2-q_{x}\right|-\left|1 / 4-q_{z}\right|$ for $T$ near $T_{c}$ for various RMO's. Positive $\Pi$ favors locking $q_{x}$ to the value $1 / 4$ in preference to locking $q_{x}$ to the value $1 / 2$.

\begin{tabular}{||c|ccccc||}
\hline $\mathrm{R}=$ & $\operatorname{Tm}[28]$ & $\operatorname{Er}[26]$ & $\mathrm{Y}[51,34]$ & $\mathrm{Ho}[29]$ & $\mathrm{Tb}[27,24]$ \\
\hline$q_{z}=$ & 0.252 & 0.244 & 0.255 & 0.237 & 0.277 \\
$q_{x}=$ & 0.472 & 0.479 & 0.482 & 0.488 & 0.487 \\
$\Pi=$ & 0.026 & 0.015 & 0.013 & -0.001 & -0.010 \\
\hline
\end{tabular}

of secondary degrees of freedom, which generate effective temperature-dependent exchange coefficients. Note that the whole diagram corresponds to the close vicinity of $\mathbf{q}=(1 / 2,0,1 / 4)$, so that this temperature dependence is relatively weak. As shown in figure 4, the real RMO's go directly from the $(I, I)_{1}$ phase into either an $(I, C)$ (for $\mathrm{R}=\mathrm{Er}, \mathrm{Tm}, \mathrm{Y}$ ) or into the $(C, C)$ phase (for $\mathrm{R}=\mathrm{Ho}$, Dy, Tb). In the former case, we now argue that this phase must be the $(I, C)_{2}$ phase: Since the experimentally observed phase is ferroelectric, it follows that there must exist two OP's, $\sigma_{a}$ and $\sigma_{b}$. Once both order parameters exist, this phase could be either $(I, I)_{2}$ or $(I, C)_{2}$. Since the experiments find that $q_{z}=1 / 4$, this must be $(I, C)_{2}$. Indeed, we conclude that the trajectories for $\mathrm{R}=\mathrm{Er}, \mathrm{Tm}, \mathrm{Y}$ are represented by the dashed lines with long dashes in the phase diagram. As the same lines indicate, one would then go into the $(C, C)$ phase, as indeed observed. At lower temperatures, the trajectories could leave the $(C, C)$ phase to the other side of the parabolic 'tongue', and enter a less commensurate phase, which could be paraelectric $\left[(I, I)_{1}\right]$ or ferroelectric $\left[(I, I)_{2}\right.$ or $(C, I)]$.

As indicated by the dashed line with short dashes in the same figure, one can also go directly from $(I, I)_{1}$ into $(C, C)$. This trajectory thus describes the RMO's with $\mathrm{R}=\mathrm{Ho}$, Dy, Tb. In the $(C, C)$ phase, which is ferroelectric, (27) indicates that $\left|\sigma_{1}\right| \neq\left|\sigma_{2}\right|$. The quartic term of (26) implies that either $\left|\sigma_{1}\right|=\left|\sigma_{2}\right|$, or one of them is zero, so that $\sigma_{1} \sigma_{2}=0$. Thus only the first term in (27) survives and it explains the observation 36, 37 that the spontaneous polarization lies along the $\mathbf{b}$ axis. Finally, we should mention that the fact that different R's follow slightly different trajectories is reasonable from the following qualitative point of view. For Tm, Er, and Y the value of $q_{x}$ (listed in table 2) is much closer to $1 / 4$ and therefore is more likely to be locked to $q_{x}=1 / 4$ than is that of Ho and Tb.

For DyMO, experiments have not definitively determined the sequence of phase transitions in the wave vector, because the large incoherent neutron cross section of the Dy nucleus causes experimental problems. A recent X-ray experiment 40 has confirmed the existence 39 of the $(C, C)$ state. The specific heat 37. provides evidence that there is a single intermediate phase between this state and the paramagnetic state. As argued in connection with figure 5 , this intermediate phase has to be an $(I, I)_{1}$ phase, because we do not allow the possibility of accidentally hitting the multicritical point where the $\mathrm{P}$ phase meets the $(I, C)$ (in figure $5 \mathrm{a}$ ) or $(C, I)$ phase (in figure $5 \mathrm{~b}$ ). This proposed phase exhibits a single OP, which is also consistent with the fact that DyMO is paraelectric for $T>40 \mathrm{~K}$ (see figure 4 ).

We now return to the phase diagram of figure 5. All the RMO's have q close to $(1 / 2,0,1 / 4)$ (see table 2), so they leave the $\mathrm{P}$ phase near the apex of the tongue of 
figure 6a or 6b. The effects of a magnetic field are explained as follows: it generates magnetic moments on the $\mathrm{R}$ ions (even above their ordering temperature). Since these ions couple to the $\mathrm{Mn}$ ions, their moment changes the effective Mn-Mn interactions, thus changing the 'control parameters' and the optimal q. This often moves the material towards the $(C, C)$ tongue, resulting in a transition from $(I, C)((I, I)$ when paraelectric) back into the CM phase [32, 29]. Pressure[52] has similar effects.

\subsection{Spin structures in the $(C, C)$ phase}

The introduction of OP's leads to a natural interpretation of neutron scattering results for the $(C, C)$ phase in YMO. Figure 7 shows the $\mathrm{Mn}^{3+}$ a-b plane spin components in the CM phase of YMO, from the neutron diffraction results of [35] 2 and [25]. These two structures are obviously similar, and one might ask what symmetry (if any) relates them. (This degeneracy was also found in the first-principles calculation of [53.) We now show that these two structures are indeed equivalent. 21] To identify the symmetry element that relates them note that the structure on the left is even under the glide operation $m_{x}$, while that on the right is odd under $m_{x}$. (Here one should note that spin, being a pseudovector, transforms with an additional minus sign under a mirror operation.) Then (25) indicates that the structure on the left has $\sigma_{2}=0$, whereas that on the right has $\sigma_{1}=0$. Going between these two structures corresponds to a rotation in OP space. This equivalence is easily understood when OP's are introduced, as done here. Since either $\sigma_{1}=0$ or $\sigma_{2}=0$, we conclude from the discussion below (26), that $w+2 v-2|v|$ is positive and both OP's can not order simultaneously [6, 21]. This conclusion supports that reached above, namely that since the CM phase is ferroelectric, the fourth order terms in (26) must select $\sigma_{1} \sigma_{2}=0$.

Figure 7. (Color online) Schematic diagram of the $\mathbf{a}$ and $\mathbf{b}$ components of the $\mathrm{Mn}^{3+}$ spins in a single a-b plane of YMO for the CM phase. The glide $m_{x}$ consists of a mirror plane $\mathrm{M}$ at $x=a / 4$ followed by a translation $\mathrm{b} / 2$ along $y$. Left: the structure given in table III of [25] (with the c-components not shown). Right: the structure given in figure 2 of 35 (who reported zero c-components of spin.)

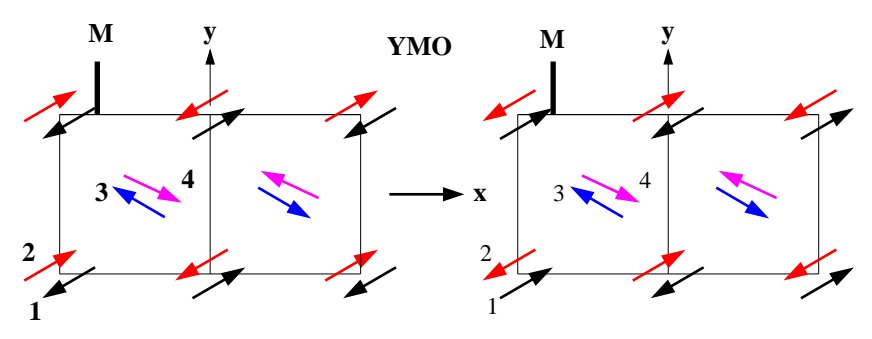

To make this identification more quantitative, we consider the magnetic structure which H. Kimura et al. 25] deduced from their neutron diffraction study, which we summarize in table 3. Their structure determination was based on an unrestricted fit, in which no particular symmetry was assumed. In contrast, our analysis based on representation theory assumes that the magnetic structure is characterized by the two complex-valued order parameters $\sigma_{1}$ and $\sigma_{2}$, with corresponding spin wave functions

* The top (bottom) panel of figure 2 in this paper should be labeled $24.7 \mathrm{~K}(1.9 \mathrm{~K})$. 
Table 3. Magnetic structures of $\mathrm{YMn}_{2} \mathrm{O}_{5}$ at $T=25 \mathrm{~K}$ showing the spins vectors of the eight $\mathrm{Mn}^{3+}$ and the eight $\mathrm{Mn}^{4+}$ sites within the cell $a_{0} \times b_{0} \times 2 c_{0}$. The complete magnetic unit cell is found by antiferromagnetically doubling the cell in both the $\mathbf{a}$ and $\mathbf{c}$ directions.

\begin{tabular}{|c|c|c|c|c|c|c|c|c|c|c|c|c|}
\hline \multicolumn{6}{|c|}{$\mathrm{Mn}^{3+}$} & & \multicolumn{6}{|c|}{$\mathrm{Mn}^{4+}$} \\
\hline$\overline{M_{x}}$ & $\overline{M_{y}}$ & $\overline{M_{z}}$ & $M_{x}$ & $\overline{M y}$ & $\overline{M_{z}}$ & $n$ & $M_{x}$ & $M_{y}$ & $M_{z}$ & $M_{x}$ & $M_{y}$ & $M_{z}$ \\
\hline H. & imura & al. & & his wo & & & H. & imura & $a l$. & & his wo & \\
\hline-2.02 & -0.41 & -0.71 & -2.04 & -0.38 & -0.67 & 1 & 1.74 & 0.51 & 0.28 & 1.72 & 0.55 & 0.30 \\
\hline 2.20 & -0.40 & -0.24 & 2.18 & -0.41 & -0.26 & 2 & 1.69 & -0.59 & -0.32 & 1.72 & -0.55 & -0.30 \\
\hline-2.06 & 0.35 & 0.63 & -2.04 & 0.38 & 0.67 & 3 & 0.98 & -0.33 & -0.69 & 0.99 & -0.30 & -0.63 \\
\hline-2.15 & -0.42 & -0.29 & -2.18 & -0.41 & -0.26 & 4 & 1.00 & 0.27 & 0.57 & 0.99 & 0.30 & 0.63 \\
\hline 2.82 & 0.58 & -0.51 & 2.85 & 0.53 & -0.48 & 5 & -1.65 & -0.46 & 0.51 & -1.63 & -0.50 & 0.56 \\
\hline-3.07 & 0.55 & -0.18 & -3.04 & 0.57 & -0.19 & 6 & -1.61 & 0.55 & -0.62 & -1.63 & 0.50 & -0.56 \\
\hline 2.87 & -0.49 & 0.45 & 2.85 & -0.53 & 0.48 & 7 & -2.12 & 0.74 & -0.09 & -2.15 & 0.68 & -0.10 \\
\hline 2.99 & 0.59 & -0.21 & 3.04 & 0.57 & -0.19 & 8 & -2.18 & -0.63 & 0.09 & -2.15 & -0.68 & 0.10 \\
\hline
\end{tabular}

which are given in [6], but more conveniently in table IX of [41. Since we expect that $\sigma_{1} \sigma_{2}=0$, our theory would imply that the spin structure should be fitted with only one OP component. Indeed, we find that Kimura et al.'s data can be fitted with $\sigma_{2}=0$. Optimizing the parameters of table IX of [41] so as to reproduce the spin structure of Kimura et al., we found the optimal structure constants to be

$$
\begin{aligned}
& \mathbf{r}_{1}=(-0.387,-0.072,0.091 i), \quad \mathbf{r}_{2}=(0.413,0.078,0.036 i) \\
& \mathbf{z}=(0.257+0.049 i,-0.081-0.017 i, 0.031-0.063 i) .
\end{aligned}
$$

With the normalization $2\left|\mathbf{r}_{1}\right|^{2}+2\left|\mathbf{r}_{2}\right|^{2}+4|\mathbf{z}|^{2}=1$, the complex-order parameter was found to be

$$
\sigma_{1}=5.2698+i 7.3691 .
$$

(This complex phase can not be explained by a low order anisotropy in the complex $\sigma_{1}$ plane.) From table 3 one sees that the structure assuming the validity of representation theory is quite close to that of the unrestricted fit of Kimura et al.. The difference between these two structures is that our version respects the symmetry one would attribute to a structure having only $\sigma_{1}$ nonzero. Thus, in our structure the magnetic sublattices are related in pairs, whereas in the structure of [25] these sublattices are almost, but not exactly, related. To characterize the difference between these two structures, note that $\left|\sigma_{1}\right| \approx 9.1$ gives the square root of the sum of the squares of the spin amplitudes within the cell of table 3. The analogous quantity for the difference vector between the two structures is 0.23 , indicating that the difference, if real, corresponds to an additional order parameter whose magnitude is about $2.5 \%$ of $\sigma_{1}$. As we explained, near the high-temperature limit of this phase one can only have either $\left|\sigma_{1} \sigma_{2}\right|=0$ or $\left|\sigma_{1}\right|=\left|\sigma_{2}\right|$. Thus, if $\left|\sigma_{2}\right| \neq 0$ then we would expect it to be of the same order as $\left|\sigma_{1}\right|$. Thus, it seems unlikely that if such an additional order parameter would emerge, it would be so small deep in the CM phase, where the data were taken. Accordingly, we propose that the actual magnetic structure in the $(C, C)$ phase of YMO corresponds to a single order parameter $\sigma_{1}$. We have also identified that the data from 25] on $\mathrm{HoMn}_{2} \mathrm{O}_{5}$ exhibit the same symmetry: namely the $(C, C)$ phase is characterized by the single order parameter $\sigma_{1}$. Similarly, we identify that the magnetic structure of the $\mathrm{Mn}$ spins in $\mathrm{ErMn}_{2} \mathrm{O}_{5}$, as reported in [25], is also consistent with the symmetry associated with the single order parameter $\sigma_{1}$. However, the phases $\phi_{x}$ of the $x$-components of the Er magnetic moments $(0.8 \pi$ and $-0.3 \pi)$ do not agree with the values ( $\pi$ or 0 ) corresponding to $\sigma_{1}$. It would be interesting to 
check the sensitivity of the data to variation of these phases. It is interesting that the structures of all the 125's determined in 25 , have $\sigma_{2}=0$, even though the structure with $\sigma_{1}=0$ represents an equivalent way that magnetic ordering can break symmetry. Apparently, the sample preparation (which might create some uniaxial strain) or some other experimental detail (stray electric fields?) chooses the structure with $\sigma_{2}=0$ in these experiments. It would be interesting to study the cause for this apparent symmetry breaking.

The selection of which OP is nonzero in the $(C, C)$ phase is a result of broken symmetry. An electric field along $b$ would order $P_{b}$, and then (15) would select either $\sigma_{1}$ or $\sigma_{2}$, depending on the sign of the field. Therefore we suggest cooling the sample into the FE phase in the presence of a small electric field along $b$. Depending on the sign of the electric field one should get either the left- or the right-hand panel of figure 7] This was indeed confirmed experimentally [55]. (A similar experiment was recently performed in $\mathrm{TbMnO}_{3}[56]$ ).

\section{Critical phenomena}

All the quantitative results presented above were based on the Landau expansion and on mean field theory. Although these theories usually give reasonable predictions far away from critical points, fluctuations must be included in the critical regimes. We start with $\mathrm{NVO}$ and $\mathrm{TbMnO}_{3}$. In these materials, one first goes from the $\mathrm{P}$ phase into the HTI phase, which is represented by a single complex OP $\sigma_{\text {HTI }}$. Since the free energy only involves $\left|\sigma_{\mathrm{HTI}}\right|^{2}$, it does not depend on the phase of this complex number, and therefore this transition belongs to the universality class of the XY model, with the critical exponents of an isotropic $(n=2)$-component spin model. The transition from the HTI phase into the LTI phase, at $T_{<}$, is also continuous. A priori, $\sigma_{\mathrm{LTI}}$ is also a complex number, which would be described by an XY model. However, as we discussed after (21), terms like $\left[\left(\sigma_{\mathrm{HTI}} \sigma_{\mathrm{LTI}}^{*}\right)^{2}+\right.$ c.c. $]$ would lock the wave vectors of the two order parameters to each other, even before one reaches $T_{<}$. This lock-in is indeed observed experimentally in the LTI phases of NVO 12 and $\mathrm{TbMnO}_{3}[5]$.

Technically, near $T_{<}$we have a finite order parameter $\left\langle\sigma_{\mathrm{HTI}}\right\rangle \equiv a e^{i \alpha}$. Writing also $\sigma_{\text {LTI }} \equiv e^{-i \alpha}(b+i c)$, the above locking term thus becomes $a^{2}\left(b^{2}-c^{2}\right)$. Therefore, the real order parameters $b$ and $c$ now have different quadratic terms, and only one of them (depending on the sign of the overall coefficient) orders at a temperature slightly above the 'bare' $T_{<}$. As stated above, the fixed length constraint prefers $\sigma_{\mathrm{HTI}}$ and $\sigma_{\text {LTI }}$ to have different phases, which implies that $c$ orders first, and the phases of the two order parameters differ by $\pi / 2$. This then yields a helical structure in the LTI phase [15, 16, 12. Furthermore, this phase relation is also confirmed by the existence of a ferroelectric moment in the LTI phase, which would not exist if $\phi_{\mathrm{HTI}}=\phi_{\mathrm{LTI}}$ (namely if $b$ were to order, rather than $c$ ), see (14). Thus, the transition from HTI to LTI belongs to the Ising $(n=1)$ universality class. Further away from the critical point the critical exponents may approach their mean field values $\gamma=1$ and $\beta=1 / 2$.

We next consider the ME interaction, (12) and (14). Assuming that indeed only $c$ orders, we find that near the HTI $\rightarrow$ LTI transition one can replace (14) by

$$
V_{\text {int }}=2 r_{b} a c P_{b} \text {. }
$$

This immediately implies that the actual order parameter at this transition is not just $c$, but rather a linear combination of $c$ and $P_{b}$ [54, 6]. This implies that the dielectric constant should diverge near $T_{<}$, as $\epsilon_{b} \sim\left|T-T_{<}\right|^{-\gamma}$, with the Ising susceptibility 
exponent $\gamma$. However, as noted before (12), $\chi_{E}^{-1}$ is much larger than $\left|T-T_{<}\right|$, and therefore the amplitude of this divergent term (related to the amplitude of $P_{b}$ in the mixed OP) can be quite small. It would be useful to search for this divergence experimentally. Similarly, we expect that both $c$ and $P_{b}$ grow below $T_{<}$as $\left(T_{<}-T\right)^{\beta}$, with the Ising order parameter exponent $\beta$.

We next turn to RFMO. As discussed in Sec. 2.3, the ordered phase has two complex components of the magnetic OP, $\sigma_{1}$ and $\sigma_{2}$, and therefore altogether we have $n=4$ OP components, as described by (10). In fact, this free energy can be written as

$$
\begin{aligned}
F & =\left(T-T_{c}\right)\left(\left|\sigma_{1}\left(q_{z}\right)\right|^{2}+\left|\sigma_{2}\left(q_{z}\right)\right|^{2}\right)+u\left(\left|\sigma_{1}\left(q_{z}\right)\right|^{4}+\left|\sigma_{2}\left(q_{z}\right)\right|^{4}\right) \\
& +\tilde{v}\left|\sigma_{1}\left(q_{z}\right)\right|^{2}\left|\sigma_{2}\left(q_{z}\right)\right|^{2} .
\end{aligned}
$$

This can be viewed as the free energy of two XY models (with OP's $\sigma_{1}$ and $\sigma_{2}$ ), which are coupled by the last term. In terms of the renormalization group (RG), this model has two competing fixed points: the isotropic $(n=4)$ one with $\tilde{v}=2 u$, and the decoupled one with $\tilde{v}=0$ [57. It turns out that $v$ is slightly relevant near the isotropic fixed point, and $\tilde{v}$ is slightly irrelevant near the decoupled fixed point, so that as $T$ approaches $T_{<}$one could follow two scenarios. If $v=\tilde{v}-2 u<0$, iteration would make it more negative, and one could end up with a crossover from the isotropic $(n=4)$ critical behavior to the asymptotic behavior of two decoupled $\mathrm{XY}$ models. However, this crossover is very slow. Therefore, one might either observe effective exponents close to those of the isotropic $(n=4)$ critical behavior, or one might encounter relatively large corrections to the decoupled critical behavior, due to the irrelevant parameter $\tilde{v}$, which would be renormalized into $\tilde{v}\left(T_{<}-T\right)^{-\alpha}$, where $\alpha$ is the specific heat exponent of the XY model. Alternatively, if $v>0$ then $v$ would grow larger under iterations, and one would never reach the vicinity of the stable fixed point at $\tilde{v}=2 u+v=0$. In this case, one probably ends up with a slow crossover to a weak first order transition.

The ME interaction in RFMO is given in (15). Thus, $P_{c} \sim\left\langle\left|\sigma_{1}\left(q_{z}\right)\right|^{2}-\left|\sigma_{2}\left(q_{z}\right)\right|^{2}\right\rangle$. The RHS of this relation represents an order parameter anisotropy. Near the isotropic fixed point, this average scales as

$$
P_{c} \sim\left\langle\left|\sigma_{1}\left(q_{z}\right)\right|^{2}-\left|\sigma_{2}\left(q_{z}\right)\right|^{2}\right\rangle \sim\left\langle\left|\sigma_{1}\right|^{2}\right\rangle^{\lambda},
$$

where the exponent $\lambda>1$ is associated with the scaling of quadratic anisotropy terms near the isotropic $n=4$ fixed point [58. However, for this result to hold we must have $\sigma_{1} \sigma_{2}=0$, which arises only if $v>0$. As explained above, in this case we expect a crossover to a weak first order transition. Thus, as $T$ is increased towards $T_{<}$we would expect a gradual variation from the mean field result, $P_{c} \sim\left\langle\left|\sigma_{1}\right|^{2}\right\rangle$, via the critical behavior of (37), to a weak first order transition. The mean field behavior, with $\lambda=1$, implies that the FE moment is proportional to the intensity of Bragg peaks, as apparently found experimentally [18. It would be interesting to check this relation close to $T_{<}$.

Finally we turn to RMO. As stated, the ordering below the $\mathrm{P}$ phase is into the $(I, I)_{1}$ phase, which corresponds to a single irrep, say $\Gamma_{a}$. As seen from (18), this ordering involves the two complex OP's $\sigma_{a}^{+}$and $\sigma_{a}^{-}$, and therefore belongs to some $n=4$ universality class. In the $(I, I)_{1}$ phase, where $q_{z} \neq 1 / 4$, the quartic terms in the free energy include only those with the coefficients $c_{1}$ and $c_{2}$. Clearly, this free energy is equivalent to the one discussed above for RFMO, yielding only one wave vector if $c_{2}>0$ and two wave vectors if $c_{2}<0$. In the former case one probably flows under 
the RG towards a weak first order transition, while in the latter case one would flow towards the stable decoupled fixed point. Thus, the question whether one or two wave vectors order is directly related to the nature of the critical behavior.

The situation changes in the $(I, C)_{1}$ phase, where one also needs to include the Umklapp term with $c_{3}$. Near the decoupled fixed point, this term involves products of anisotropies in each of the XY models, and thus it can be shown to be relevant [57. As far as we know, this free energy has no stable fixed point, and one would eventually end up with a weak first order transition. However, in the vicinity of the isotropic fixed point, where $c_{2}$ and $c_{3}$ are small, one could still observe the critical exponents of the isotropic $n=4$ universality class. In any case, in the generic case the phase $(I, C)_{1}$ is reached from the phase $(I, I)_{1}$ via a first order transition, so that the critical behavior of the former can only be expected near the multicritical point where $J_{z}=J_{z c}$.

We next discuss the transition into the (so far unobserved) $(I, I)_{2}$ phase. We start with the simple case, where only $\sigma_{a}^{+}$orders in the $(I, I)_{1}$ phase. As explained after (22), the locking of the wave vectors of $\sigma_{a}^{+}$and $\sigma_{b}^{+}$fixes the phase of $\sigma_{b}^{+}$, so that the transition into the $(I, I)_{2}$ phase now involves an Ising-like order parameter. The situation now becomes exactly the same as in (35): the dielectric constant $\epsilon_{b}$ would diverge with the Ising exponent $\gamma$, and $P_{b}$ would grow in the $(I, I)_{2}$ phase with the Ising exponent $\beta$.

The transition from $(I, I)_{1}$ into $(I, C)_{2}$ is also weakly first order, since it involves a lock-in of $q_{z}$. However, if the discontinuity is small (as seems to be the case experimentally), we can still discuss criticality of the OP's associated with $\Gamma_{b}$. As discussed after (22), this ordering should also belong to the Ising universality class: before one reaches this transition one should see $\epsilon_{b} \sim\left|T-T_{c b}^{\prime}\right|^{-\gamma}$ and $P_{b} \sim\left(T_{c b}^{\prime}-T\right)^{\beta}$, with Ising exponents. Since $\Delta$ now introduces several additional quadratic terms in the $\sigma_{b}$ 's, this transition is expected to occur at a temperature $T_{c b}^{\prime}$ higher than $T_{c b}$, where one would have the $(I, I)_{1} \rightarrow(I, I)_{2}$ continuous transition.

Near the $\mathrm{P} \rightarrow(I, I)_{1}$ transition (which occurs at $T_{C 1}$ ), a leading fluctuation expansion yields $\Delta \epsilon \propto\left\langle P_{b}^{2}\right\rangle \propto\left|\left\langle\sigma_{a}^{2}\right\rangle\left\langle\sigma_{b}^{2}\right\rangle\right|$. Since only $\sigma_{a}$ becomes critical there, we expect singularities in $\epsilon$ which behave as the energy $\left(\left|T-T_{C 1}\right|^{1-\alpha}\right)$ and as the square of the OP $\left(\left(T_{C 1}-T\right)^{2 \beta}\right)$, but with the appropriate effective $n=4$ exponents. Indeed, experiments[52] show a break in slope at $T_{C 1}$, apparently confirming this prediction. This behavior is also expected for other multiferroics and indeed this may explain the anomaly seen in the dielectric constant of NVO shown in Fig. 4b of [22. In addition, this anomaly in the zero frequency dielectric constant reflects the emergence of a resonance in the frequency-dependent dielectric constant due to electromagnons 44, 45, 46.

\section{Summary}

We have developed a phase diagram to explain the multiferroic behavior of the family of 125 's systems and have proposed several experiments to explore the unusual symmetries of these systems. In view of our current understanding it seems unnecessary to invoke the alternate route to multiferroicity proposed in [59], particularly as a microscopic calculation 35 , having exactly the symmetry we have invoked reproduces the experimental data for $\mathrm{YMn}_{2} \mathrm{O}_{5}$ quite well. 
Order Parameters and Phase Diagrams of Multiferroics

\section{Acknowledgments}

We thank M. Kenzelmann and S. H. Lee for helpful interactions. AA and OEW acknowledge support from the ISF.

* Also emeritus, Tel Aviv Univesity.

\section{References}

[1] L. D. Landau and I. M. Lifshitz, Statistical Physics (Pergamon, 1978), Sec. 139.

[2] E. F. Bertaut, Journal de Physique, Colloque C1, 32462 (1971).

[3] J. Rossat-Mignod, in Methods of Experimental Physics, Chap. 20: Magnetic Structures, ed. K. Skold and D. L. Price, Vol. 23, p69 (Academic Press, 1987).

[4] G. Lawes, A. B. Harris, T. Kimura, N. Rogado, R. J. Cava, A. Aharony, O. Entin-Wohlman, T. Yildirim, M. Kenzelmann, C. Broholm, and A. P. Ramirez, Phys. Rev. Lett. 95, 087205 (2005).

[5] M. Kenzelmann, A. B. Harris, S. Jonas, C. Broholm, J. Schafer, S. B. Kim, C. L. Zhang, S.-W. Cheong, O. P. Vajk, and J. W. Lynn, Phys. Rev. Lett. 95, 087206 (2005).

[6] A. B. Harris, Phys. Rev. B 76, 054447 (2007). Erratum, Phys. Rev. B 77, 019901 (2008).

[7] A. P. Cracknell, J. Phys. C. 4, 2488 (1971); D. B. Litvin and W. Opechowski, Physica 76, 538 (1974); Yu. A. Izyumov, V. E. Naish and R. P. Ozerov, Neutron Diffraction of Magnetic Materials (Springer-Verlag, Amsterdam, 1991).

[8] J. Schweizer, J. Villain, and A. B. Harris, Eur. J. Phys. - Appl. Phys. 38, 31 (2007)

[9] P. G. Radaelli and L. C. Chapon, Phys. Rev. B 76, 054428 (2007).

[10] A. J. C. Wilson, International Tables for Crystallography (Kluwer Academic, Dordrecht, 1995) Vol. A.

[11] G. Lawes, M. Kenzelmann, N. Rogado, K. H. Kim, G. A. Jorge, R. J. Cava, A. Aharony, O. Entin-Wohlman, A. B. Harris, T. Yildirim, Q. Z. Huang, S. Park, C. Broholm, and A. P. Ramirez, Phys. Rev. Lett. 93, 247201 (2004).

[12] M. Kenzelmann, A. B. Harris, A. Aharony, O. Entin-Wohlman, T. Yildirim, Q. Huang, S. Park, G. Lawes, C. Broholm, N. Rogado, R. J. Cava, K. H. Kim, G. Jorge, and A. P. Ramirez, Phys. Rev. B 74, 014429 (2006).

[13] A. B. Harris, J. Appl. Phys. 99, 08E303 (2006).

[14] T. Kimura, G. Lawes, T. Goto, Y. Tokura, and A. P. Ramirez, Phys. Rev. B 71, 224425 (2005).

[15] T. Kaplan, Phys. Rev. 124, 329 (1961).

[16] T. Nagamiya, in Solid State Physics, ed. F. Seitz and D. Turnbull (Academic, New York, 1967), Vol. 20, p346.

[17] L. E. Svistov, A. I. Smirnov, L. A. Prozorova, O. A. Petrenko, L. N. Demianets, and A. Y. Shapiro, Phys. Rev. B 67, 094434 (2003).

[18] M. Kenzelmann, G. Lawes, A. B. Harris, G. Gasparovic, C. Broholm, A. P. Ramirez, G. A. Jorge, M. Jaime, S. Park, Q. Huang, A. Ya. Shapiro, and L. N. Demianets, Phys. Rev. Lett. 98, 267205 (2007).

[19] R. Kajimoto, H. Yoshizawa, H. Shintani, T. Kimura, and Y. Tokura, Phys. Rev. B 70012401 (2004); 70, 219904(E) (2004).

[20] G. Gasparovic, Ph. D. Thesis, Johns Hopklins University, 2004.

[21] A. B. Harris, A. Aharony, and O. Entin-Wohlman, arXiv:0802.0604

$[22]$ G. Lawes, M. Kenzelmann, and C. Broholm, in this volume, see figure 6.

[23] I. A. Sergienko, C. Sen, and E. Dagotto, Phys. Rev. Lett. 97, 227204 (2006).

[24] L. C. Chapon, G. R. Blake, M. J. Gutmann, S. Park, N. Hur, P. G. Radaelli, and S.-W. Cheong, Phys. Rev. Lett. 93, 177402 (2004).

[25] H. Kimura, S. Kobayashi, Y. Fukuda, T. Osawa, Y. Kamada, Y. Noda, I. Kagomiya, and K. Kohn, J. Phys. Soc. Jpn. 76, 074706 (2007).

[26] S. Kobayashi, T. Osawa, H. Kimura, Y. Noda, I. Kagomiya, and K. Kohn, J. Phys. Soc. Jpn. 73, 1031 (2004).

[27] S. Kobayashi, T. Osawa, H. Kimura, Y. Noda, N. Kasahara, S. Mitsuda, and K. Kohn, J. Phys. Soc. Jpn. 73, 3439 (2004).

[28] S. Kobayashi, H. Kimura, Y. Noda, and K. Kohn 2005, J. Phys. Soc. Jpn. 74, 468 (2005). 
[29] H. Kimura, Y. Kamada, Y. Noda, K. Kaneko, N. Metoki, and K. Kohn, J. Phys. Soc. Jpn. 75, 113701 (2006).

[30] A. Inomata and K. Kohn, J. Phys. Condensed Matter 8, 2673 (1996).

[31] I. Kagomiya, S. Matsumoto, K. Kohn, Y. Fukuda, T. Shoubu, H. Kimura, Y. Noda, and N. Ikeda, Ferroelectrics 286, 167 (2003).

[32] D. Higashiyama, S. Miyasaka, and Y. Tokura, Phys. Rev. B 72, 064421 (2005).

[33] M. Uga, N. Iwata, and K. Kohn, Ferroelectrics 219, 691 (1998).

[34] S. Kobayashi, T. Osawa, H. Kimura, Y. Noda, I. Kagomiya, and K. Kohn, J. Phys. Soc. Jpn. 73, 1593 (2004).

[35] L. C. Chapon, P. G. Radaelli, G. R. Blake, S. Park, and S.-W. Cheong, Phys. Rev. Lett. 96, 097601 (2006).

[36] N. Hur, S. Park, P. A. Sharma, J. S. Ahn, S. Guha, and S.-W. Cheong, Nature 429, 392 (2004).

[37] N. Hur, S. Park, P. A. Sharma, S. Guha, and S.-W.Cheong, Phys. Rev. Lett. 93, 107207 (2004).

[38] D. Higashiyama, S. Miyasaka, N. Kida, T. Arima, and Y. Tokura, Phys. Rev. B 70, 174405 (2004).

[39] W. Ratcliff II, V. Kiryukhin, M. Kenzelmann, S.-H. Lee, R. Erwin, J. Schefer, N. Hur, S. Park, and S.-W. Cheong, Phys. Rev. B 72, 060407(R) (2005).

[40] R. A. Ewings, A. T. Boothroyd, D. F. McMorrow, D. Mannix, H. C. Walker, and B. M. R. Wanklyn, arXiv:0711.1745

[41] A. B. Harris, M. Kenzelmann, A. Aharony, and O. Entin-Wohlman, arXiv:0803.0945.

[42] S. Skanthakumar, J. W. Lynn, J. L. Peng, and Z. Y. Li, Phys. Rev. B 47, 6173 (1993).

[43] G. R. Blake, L. C. Chapon, P. G. Radaelli, S. Park, N. Hur, S.-W. Cheong, and J. RodriguezCarvajal, Phys. Rev. B 71, 214402 (2005).

[44] A. Pimenov, A. A. Mukhin, V. Yu. Ivanov, V. D. Travkin, A. M. Balbashov, and A. Loidl, Nature Phys. 2, 97 (2006).

[45] R. Valdes Aguilar, A. B. Sushkov, C. L. Zhang, Y. J. Choi, S.-W. Cheong, and H. D. Drew, Phys. Rev. B 76, 060404 (2007).

[46] A. B. Sushkov, R. Valdes Aguilar, S. Park, S.-W.Cheong, and H. D. Drew, Phys. Rev. Lett. 98, 027202 (2007).

[47] T. Kimura, T. Goto, H. Shintani, K. Ishizaka, T. Arima, and Y. Tokura, Nature 426, 55 (2003).

[48] C. Beutier, A. Bombardi, C. Vecchini, P. G. Radaelli, S. Park, S-W. Cheong and L. C. Chapon, arXiv:0803.3779

[49] B. Mihailova, M. M. Gospodinov, B. Guttler, F. Yen, A. P. Litvinchuk, and M. N. Iliev, Phys. Rev. B 71, 172301 (2005).

[50] T. Yildirim, A. B. Harris, A. Aharony, and O. Entin-Wohlman, Phys. Rev. B 73, 184433 (2006).

[51] I. Kagomiya, H. Kimura, Y. Noda, and K. Kohn, J. Phys. Soc. Jpn. 70, Suppl. A 145 (2001).

[52] C. R. dela Cruz, B. Lorenz, Y. Y. Sun, Y. Wang, S. Park, S.-W. Cheong, M. M. Gospodinov, and C. W. Chu, cond-mat/0707.0318.

[53] C. Wang, G.-C. Guo, and L. He, Phys. Rev. Lett. 99, 177202 (2007).

[54] S. Goshen, D. Mukamel, H. Shaked, and S. Shtrikman, Phys. Rev. B 2, 4679 (1970).

[55] After we announced [21, we were informed about experiments by P. G. Radaelli, L. C. Chapon, A. Daoud-Aladine, C. Vecchini, P. J. Brown, T. Chatterjii, S. Park and S-W. Cheong, arXiv: 0803.3736.

[56] Y. Yamasaki, H. Sagayama, T. Goto, M. Matsuura, K. Hirota, T. Arima, and Y. Tokura, Phys. Rev. Lett. 98, 147204 (2007).

[57] A. Aharony, in Phase Transitions and Critical Phenomena, C. Domb and M. S. Green, eds., Vol. 6 (Academic Press, NY, 1976), p. 357.

[58] A. Aharony, R. J. Birgeneau, J. D. Brock, and J. D. Litster, Phys. Rev. Lett. 57, 1012 (1986).

[59] J. J. Betouras, G. Giovannetti, and J. van den Brink, Phys. Rev. Lett. 98, 257602 (2007). 


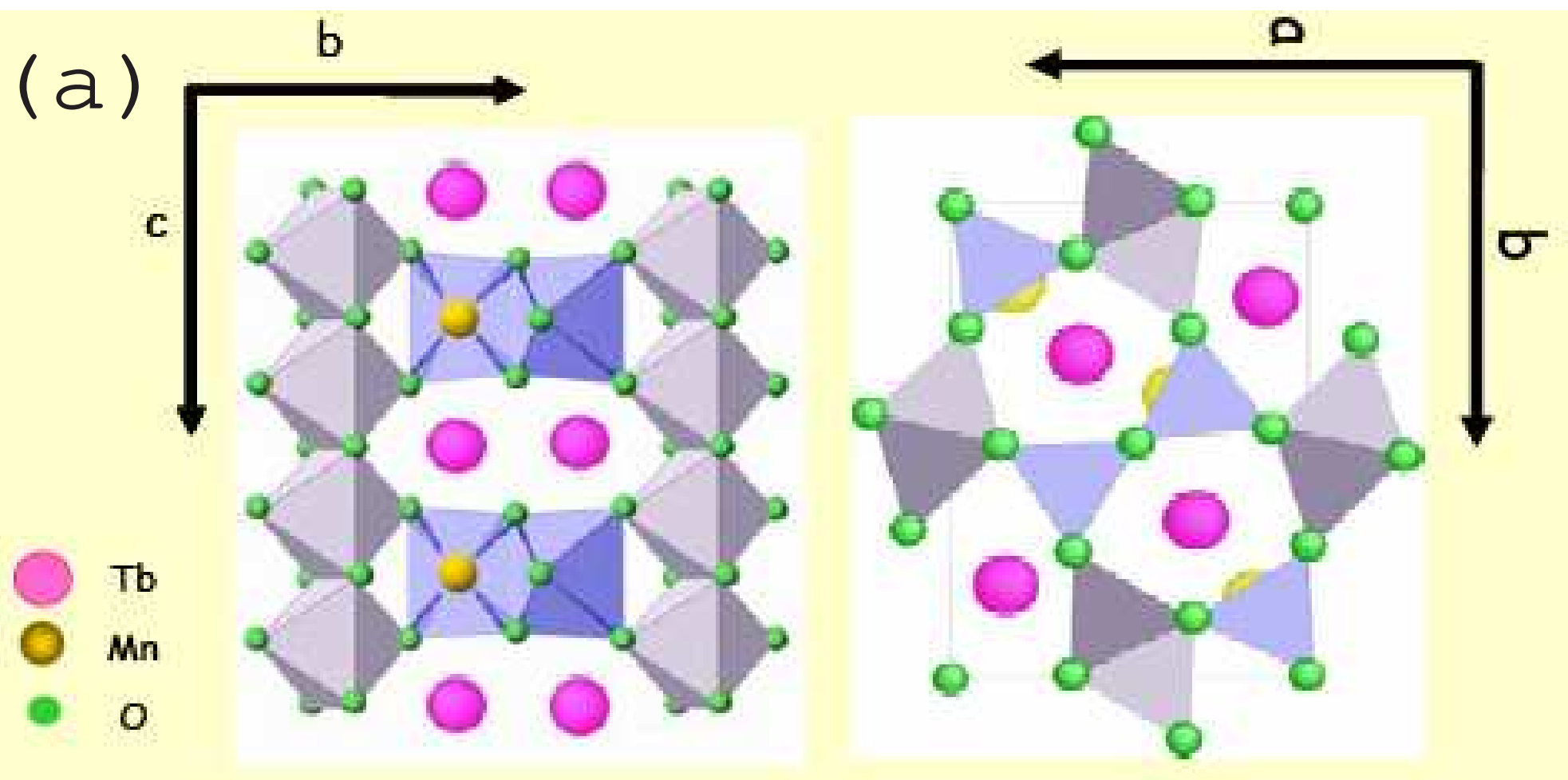


This figure "figure3.gif" is available in "gif" format from: http://arxiv.org/ps/0804.3039v1 